\title{
Competencia digital e inclusión educativa. Visiones de profesorado, alumnado y familias
}

\section{Digital competence and inclusive education. Visions of teachers, students and families}

\author{
M. Montserrat Castro Rodríguez \\ Universidade de A Coruña, A Coruña, España \\ maria.castror@udc.es \\ Diana Marín Suelves \\ Universitat de València, València, España \\ diana.marin@uv.es \\ Héctor Sáiz Fernández \\ Universitat de València, València, España \\ saizhector@alumni.uv.es
}

\begin{abstract}
Resumen
La formación de la ciudadanía en competencia digital interesa a administraciones nacionales y supranacionales. En los últimos años se han invertido ingentes cantidades económicas en la dotación de infraestructura y en I+D+I para el desarrollo tecnológico en distintos sectores de la sociedad. La integración de dispositivos digitales en la sociedad ha impacto en las políticas educativas de muchos países, llevándolos a considerar la competencia digital como aspecto clave en la enseñanza obligatoria y esencial para la inclusión social. En este artículo, se presentan los resultados de una investigación realizada en cuatro centros de Educación Primaria de Valencia y Galicia, reconocidos por su trayectoria innovadora en el uso de las TIC. El objetivo es identificar y analizar las visiones que la comunidad educativa de cada escuela tiene sobre la competencia digital que está adquiriendo el alumnado participante en prácticas educativas mediadas por TIC. Para la recogida de información se empleó metodología cualitativa, específicamente entrevistas a profesorado, familias y alumnado. Los resultados reflejan modelos diferentes de trabajo y conceptualización de la competencia digital. Todos ellos reconocen su relevancia para la inclusión en la sociedad del futuro y desmitifican su rol hegemónico en el aprendizaje escolar.
\end{abstract}

Palabras clave: competencia digital, comunidad educativa, inclusión.

\begin{abstract}
The training of citizens in digital competence is of interest to national and supranational administration. In recent years enormous amounts of money have been put into the provision of facilities and I+D+I for technological development in different sectors of society. The integration of digital devices in the industry, communication and society has had a profound impact on educational policies in many countries, making them consider the digital competence as a key in the compulsory education and essential to the social inclusion. This article shows the results of a research conducted in four primary schools from Valencia and Galicia that are recognized because of their innovative trajectory in the use of ICTs. The aim is to identify and analyse the visions of the education community members from each school about the digital competence which is being acquired by the students involved in educational practices mediated by ICTs. To collect the information, it was used a qualitative method based on interviews with teachers, families and students. The results reflect different work models and concepts about the digital
\end{abstract}


competence. It is recognized that the ICTs are relevant to the inclusion in the society of tomorrow and it has been demystified their hegemonic role on school learning.

Keywords: digital competence, education community, inclusion.

\section{Introducción}

La creación de la Unión Europea implicó grandes cambios en diferentes ámbitos, como el social, económico y educativo. En este contexto, por una parte, se elaboró el Marco Estratégico para la Cooperación Europea, en el que se establecieron como prioridades para el ciclo 2015-2020: la inclusión, la equidad, la igualdad, la no discriminación y la promoción de valores cívicos. Concretamente, la inclusión implica la oferta de una educación equitativa desde la perspectiva de la justicia social que, de acuerdo con Arnaiz (2012), contribuya al desarrollo de la cohesión social, lo que permitirá el progreso de la sociedad hacia la paz, la libertad y la justicia social. La inclusión educativa y la justicia social en los centros escolares (Tedesco, 2005) se vislumbra como la clave para construcción de una sociedad inclusiva (Gewerc \& Fraga-Varela, 2019). Por otra parte, se reformularon los planes de estudio para dar respuesta a la necesidad de diseñar el currículum en base a las competencias que permitirán un desarrollo integral del alumnado y la preparación para la vida social.

Partiendo de la base de que la inclusión requiere además de estar, poder participar y aprender en un contexto lo menos restrictivo posible, de acuerdo con lo establecido por el CAST (2011) y descrito por Alba (2016), se define el Diseño Universal para el Aprendizaje (DUA) como el conjunto de principios para el desarrollo del currículum que proporcionen a todos los estudiantes igualdad de oportunidades para aprender. En la sociedad actual, denominada sociedad red (Castells, 1998), las tecnologías permiten el abordaje de estas cuestiones, teniendo en cuenta que la propia legislación educativa considera la competencia digital (en adelante CD) como fundamental o clave. En el 2006, en el marco de las recomendaciones del Parlamento Europeo, se entiende que tener CD: "[...] entraña el uso seguro y crítico de las tecnologías de la sociedad de la información (TSI) para el trabajo, el ocio y la comunicación. Se sustenta en las competencias básicas en materia de TIC" (Recomendación del Parlamento Europeo, 2006, p.15). Posteriormente, en 2013 desde la Comisión Europea se publicará The Digital Competence Framework for Citizens, conocida como DigCom, y en 2016, DigComp 2.0: The Digital Competence Framework for Citizens, donde se explica el modelo de referencia para el desarrollo de la CD de la ciudadanía, identificando cuatro dimensiones: 1) áreas de la CD, 2) descriptores y títulos de cada área, 3) niveles de competencia y 4) ejemplos de conocimientos, habilidades y actitudes de cada competencia (Vuorikari, Punie, Carretero Gomez \& Van den Brande, 2016). Según las DigCom, la CD implica la adquisición de alfabetización en información y datos, comunicación y colaboración, creación de contenido digital, seguridad y resolución de problemas.

Las tecnologías de la información y la comunicación (en adelante TIC) han impactado en todos los ámbitos de la sociedad actual, incluyendo al educativo. En este también se han incorporado las tecnologías del aprendizaje y del conocimiento (en adelante TAC), en una clara relación de lo que significa ser competente digitalmente. La introducción de las tecnologías digitales en los centros escolares del contexto español es una realidad desde hace varias décadas (Area \& Hernández, 2018) y esto es el reflejo de las políticas educativas dirigidas a la integración de las tecnologías en la escuela, promovidas tanto

Competencia digital e inclusión educativa. Visiones de profesorado, alumnado y familias. $\mathrm{M}^{\mathrm{a}}$ Montserrat Castro Rodríguez, Diana Marín Suelves y Héctor Saiz Fernández.

Página 2 de 37 
desde el contexto europeo, nacional y autonómico, como desde instancias públicas y privadas.

Los continuos avances tecnológicos (Fuentes, López \& Pozo, 2019) han sido uno de los grandes cambios en los últimos tiempos, por lo que muchos han denominado esta sociedad como nueva sociedad del conocimiento y la información (Sanabria, Álvarez \& Peirats, 2017). Esto explica en nuestro país el impulso sostenido en el tiempo realizado por las Administraciones, gestores y familias (Colás, De Pablos \& Ballesta, 2018). También que, en paralelo, se hayan impulsado e implementado diferentes programas de forma aislada (Area, 2006) amparados por la autonomía de las Comunidades Autónomas. Por todo ello, no existe una única realidad en cuanto a la introducción de tecnologías en la escuela. En general, se observa la pérdida de hegemonía de los materiales didácticos en formato impreso, que durante años han sido el recurso principal utilizado en las aulas, para dar paso a una implantación progresiva de las TIC (Sánchez \& Arathoon, 2016).

La evolución de su implantación en los centros escolares y en la sociedad en general ha sido muy desigual, no sólo debido a la disponibilidad de dispositivos, sino fundamentalmente a las prácticas pedagógicas que se han desarrollado a lo largo de todas estas décadas (Céspedes \& Ballesta, 2018; Sancho-Gil \& Hernández-Hernández, 2018) y al significado de la CD. La investigación científica pone de manifiesto algunas digresiones en el proceso (Ramírez-García, Díaz \& Sánchez-Carrero, 2014): por una parte, la evolución legislativa impulsada en las últimas décadas ha desembocado en la actualidad en la consideración como competencia clave o básica en el currículum escolar a la CD (LOMCE, 2013); la investigación también pone de relieve que, en algunos casos, la adquisición de $\mathrm{CD}$ ha contribuido a una mejora en la igualdad de oportunidades a personas que han encontrado apoyos que facilitan su accesibilidad al conocimiento y la participación (Maquilón Sánchez, 2016; Selwood, Atkinson \& Black, 2005). Por otra parte, algunas investigaciones y voces procedentes de ámbitos muy dispares, visibilizan las importantes desigualdades que se detectan, que lejos de contribuir a una mayor equidad en el sistema escolar y social, la disminuye. Así se habla de brecha digital, la cual afecta a una parte de la población que se ve excluida en diferentes ámbitos como la escuela y el trabajo, quedando por tanto excluida a nivel social (Cid Fernández \& Rodríguez Rodríguez, 2007; Darcy, Yerbury \& Maxwell, 2019; Delfino, Beramendi \& Zubieta, 2019; Gonçalves et al., 2018). No han de ser obviados en este sentido los riesgos que se están detectando en la implementación de la tecnología (Musmarra, 2017).

En este artículo se presentan los resultados obtenidos en una investigación realizada en el marco del proyecto de $\mathrm{I}+\mathrm{D}+\mathrm{I}$ denominado La escuela en la sociedad digital: análisis y propuestas para la producción y uso de los contenidos digitales educativos (EDU201564593-R). Este proyecto ha sido financiado por el Programa Estatal de I+D+I Orientada a los Retos de la Sociedad, convocado por el Gobierno de España y dedicado al análisis de la producción y uso de contenidos didácticos digitales en los centros escolares. En estas líneas, se analizan las visiones de distintos agentes educativos pertenecientes a cuatro centros de Educación Primaria de la Comunidad Valenciana y de Galicia, sobre la CD que el alumnado está adquiriendo en estas escuelas. Las cuales han sido seleccionadas por su importante trayectoria innovadora en la incorporación de la tecnología.

\section{Evolución del concepto de competencia digital en la sociedad-escuela}

La evolución del significado del uso de la tecnología digital en el aula y su peso curricular hasta llegar a la actualidad, en la que la CD se considera como básica, está condicionada

Competencia digital e inclusión educativa. Visiones de profesorado, alumnado y familias. $\mathrm{M}^{\mathrm{a}}$ Montserrat 
por un importante cambio cualitativo relacionado con la visión que a lo largo de varias décadas se ha ido fraguando en la sociedad mundial (González Pérez, 2011; Lores Gómez, 2017; Sanabria \& Cepeda, 2016).

En los primeros años de la introducción de los recursos tecnológicos en las escuelas, llegaron a las aulas dispositivos que la sociedad asociaba sobre todo al ocio y tiempo libre. Se está hablando de televisores, radios, vídeos, dvds e incluso cámaras fotográficas. Esto, en algunas ocasiones, no fue bien considerado por parte de familias y cierto sector del profesorado por dudar de sus usos educativos. Dicha actitud derivaba posiblemente de que estos dispositivos eran usados principalmente con fines lúdicos y el potencial educativo ligado al desarrollo de la CD no se asociaba al manejo de un recurso de este tipo.

Sin embargo, con la llegada de las TIC, los proyectores de diapositivas y transparencias dan paso a ordenadores, tabletas, móviles, impresoras (ahora ya 3D), robots, programas y aplicaciones capaces de ser conectadas a internet, y que se asocian con nuevas perspectivas de uso de la tecnología (Jódar Marín, 2010; Colás, De Pablos \& Ballesta, 2018). Cuando muchos de estos dispositivos digitales llegan a la escuela ya están plenamente incluidos en distintos sectores de la sociedad, entre ellos el productivo, el laboral y el social. Incluso, con mucha frecuencia, se habla de que la escuela llega tarde en su incorporación. A priori, en el imaginario social, que una persona tenga $\mathrm{CD}$, entre otras muchas cuestiones que se le puedan atribuir desde los ámbitos especializados, ya no sólo se asocia al manejo del dispositivo. Conlleva otras acepciones como, por ejemplo, saber buscar información, ser críticos con la información disponible, hacer un uso responsable o aprovechar las funcionalidades que ofrecen para la vida cotidiana, como puede ser pedir una cita médica, hacer una solicitud a la administración o conseguir en la edad adulta un puesto laboral donde el uso de un ordenador u otros dispositivos es imprescindible. Por lo tanto, ser competente digitalmente ya no sólo implica saber hacer funcionar un dispositivo, sino también saber buscar las herramientas, transferir y aplicar conocimientos (Millard, Baldassar \& Wilding, 2018).

En el marco de la legislación educativa española, en la Orden ECD/65/2015, de 21 de enero, se define que "La competencia digital es aquella que implica el uso creativo, crítico y seguro de las tecnologías de la información y la comunicación para alcanzar los objetivos relacionados con el trabajo, la empleabilidad, el aprendizaje, el uso del tiempo libre, la inclusión y participación en la sociedad" (p.10). Atribuye a la CD, un rol fundamental para la alfabetización, en relación con la lectura y la escritura, teniendo en cuenta que el aprendizaje mediado por tecnología conlleva el manejo de un complejo conglomerado de lenguajes que se integran para el uso de las aplicaciones informáticas. Identifica también sus contribuciones a la gestión de la información, que afectan a los procesos de búsqueda, selección, análisis, interpretación, usos y aplicabilidad, creación, seguridad y resolución de problemas reales, en distintos contextos. Así mismo se reconocen sus posibles aportaciones a cuestiones transversales como la adquisición de autonomía, valores y actitudes, participación social, motivación, curiosidad y manejo de dispositivos de forma responsable. Se considera que una persona tiene CD cuando es capaz de gestionar información, de analizarla e interpretarla cotejándola y evaluándola, hace un uso consciente y responsable de la comunicación y de dispositivos digitales, creando contenidos, con seguridad y resuelve problemas.

Competencia digital e inclusión educativa. Visiones de profesorado, alumnado y familias. $\mathrm{M}^{\mathrm{a}}$ Montserrat 
En el ámbito de la investigación y el ensayo, la definición de CD es ampliamente abordada. No obstante, tal es la diversidad de definiciones y orientaciones epistemológicas sobre el término que, en la actualidad, parece alejada la posibilidad de llegar a una definición ampliamente aceptada por la comunidad científica y educativa como concluyen De Pablos, Colás, Conde \& Reyes (2016). Las investigaciones realizadas sobre la percepción que tienen los distintos miembros de la comunidad educativa sobre lo que identifican como su CD arrojan interesantes resultados, pero también evidencian las distintas interpretaciones de lo que se entiende por ser competente digitalmente. Es posible que la percepción o la concepción de lo que se entiende por $\mathrm{CD}$ en el caso de los usuarios, pueda estar en relación con las prácticas, experiencias vividas y formación recibida.

Mientras tanto en las escuelas el uso de la tecnología se encuentra en un complejo proceso de experimentación, de debate, donde las viejas y nuevas exigencias de lo que supone aprender y enseñar hoy, conviven con dificultades y oportunidades para enriquecer o compensar las desigualdades dentro del proceso de enseñanza y aprendizaje. Algunas familias y profesorado son conscientes de las nuevas oportunidades que ofrece la tecnología para atender a todo el alumnado, con o sin Necesidades Específicas de Apoyo Educativo (NEAE), donde la adquisición de CD puede contribuir al desarrollo integral de cada estudiante (Lozano, Castillo \& Cerezo, 2014; Tárraga, Vélez-Calvo, Lacruz-Pérez \& Sanz-Cervera, 2019). O incluso para favorecer la participación en la sociedad de todo el alumnado. Ya no solo se habla de su dimensión lúdica, sino que se va más allá y se toma en consideración también la productividad, la economía o la aparición de nuevos problemas derivados de su uso (Sánchez Montoya, 2007), lo que ha supuesto el aumento de la digitalización en muchos ámbitos de la vida cotidiana. Por tanto, el desarrollo de la CD parece primordial.

Para una parte importante de la sociedad, familias, profesorado y los distintos poderes económicos y sociales, los dispositivos digitales se constituyen como herramientas que contribuyen a la adquisición de una serie de habilidades y conocimientos que capacitan al estudiante para la adquisición del currículum escolar. Además, paralelamente, se está visualizando su especial importancia de cara al futuro como adulto, con fuerte vinculación al sistema productivo que directamente se asocia a la inclusión social en general y a la inserción en la vida social como ciudadanos. De esta forma la CD adquiere un valor destacado.

No obstante, se produce una situación paradójica a medida que se habla de integración de las TIC en las escuelas (Cabañes Martínez, 2016). Emergen voces que aluden a la brecha digital detectada y que se puede analizar desde distintos puntos de vista. Por una parte, se reconoce la brecha que se produce cuando el alumnado, o la población en general, no puede acceder a los dispositivos digitales o a la red de internet. Por otra parte, también se detecta una brecha en el uso que se hace en muchos colegios de recursos y materiales digitales. Esta última responde a modelos pedagógicos tradicionales tecnocráticos que no son capaces de dar una respuesta adecuada a las necesidades del alumnado, el cual vive en una sociedad fuertemente tecnologizada donde la alfabetización digital debe ir acompañada de conocimientos para un uso crítico, ético y responsable de los recursos y medios. Por último, se identifican nuevas formas de exclusión de las personas con discapacidad (Azorín y Arnáiz, 2013) por la desigualdad de oportunidades en cuanto al acceso a la información, conocimiento y educación a través de las TIC (Cabero, 2004). Estas situaciones cuestionan si realmente las escuelas están respondiendo de forma

Competencia digital e inclusión educativa. Visiones de profesorado, alumnado y familias. $\mathrm{M}^{\mathrm{a}}$ Montserrat Castro Rodríguez, Diana Marín Suelves y Héctor Saiz Fernández. 
adecuada al desarrollo de la CD a través de una implementación tecnológica eficiente, de forma que facilite la inclusión educativa y social de los educandos o, por el contrario, si en determinados casos se está produciendo e incluso incrementando la brecha digital, provocando incluso en ocasiones mayores desigualdades respecto a los más excluídos (Fraga \& Rodríguez, 2019). Cabe señalar que, aunque la CD y la integración tecnológica sean conceptos diferentes, están estrechamente relacionados, ya que, la integración previa de la tecnología en las aulas favorecerá el desarrollo de la CD entre los agentes implicados.

En este análisis, no se puede olvidar otros factores claves como el crecimiento de la innovación tecnológica, sus nuevos usos sociales y de forma muy especial, la ingente cantidad de información que reúne. Buscar información conlleva saber discernir cuál es la interesante, la útil para cada necesidad en concreto, la verdadera y la falsa, saber identificar qué recurso digital, qué software o qué procedimiento es el más adecuado en cada contexto. Los resultados de la investigación empiezan a constatar que los milenials, la generación $Z$ y los nativos digitales (Prensky, 2001) presentan desigualdades significativas en cuanto a las distintas dimensiones que integran la CD (Gewerc \& Lourdes Montero, 2015; Pötzsch, 2016; Gewerc \& Martínez-Piñeiro, 2019), la gestión de la tecnología, la búsqueda de información y el uso crítico de recursos y productos digitales (Hernández-Sánchez, Quijano \& Pérez, 2019; Pérez, Castro Zubizarreta \& Fandos, 2016). Cabe señalar que a estos se les ha venido otorgando la gran responsabilidad de ser los que enseñaran a hacer un uso adecuado del input tecnológico a las generaciones anteriores, cuya inmersión tecnológica se produjo a edades más avanzadas.

Por todo ello, surge la potencial necesidad entre todos los miembros de la sociedad de adquirir una solvente CD. Desde ciertos posicionamientos pedagógicos y sociológicos, se justifica la incorporación de la tecnología en la escuela obligatoria atribuyéndole además valor para contribuir a la igualdad de oportunidades que se debe promover desde la escuela sostenida con fondos públicos. Siguiendo las directrices de organismos internacionales, se apuesta por la opción 1:1, es decir, que cada estudiante pueda hacer uso cotidiano de un dispositivo digital, concretamente ordenadores portátiles por sus ventajas a la hora de transportarlos a sus casas. Pero también en muchas ocasiones se refieren a tabletas, dejando otros dispositivos como los móviles, para uso más esporádico.

En paralelo, familias y otras instituciones de las que depende la construcción de los espacios comunitarios, contribuyen a la adquisición de la $\mathrm{CD}$ incorporando los dispositivos en el hogar, en las bibliotecas y centros sociales públicos. También en instituciones y fundaciones privadas, que pretenden facilitar la accesibilidad tanto a los dispositivos físicos como a internet, inmersos en una gran red social (Steck, 2018). Todo ello, supone una diversificación de oportunidades para la adquisición de la CD que, en principio, podría facilitar la inclusión educativa y social.

En este marco general no se puede olvidar las grandes inversiones económicas que conlleva la adquisición de los recursos tecnológicos y del acceso a internet. De nuevo, se está poniendo en evidencia las diferencias contextuales que implican que se pueda facilitar o dificultar la accesibilidad, ya no solo en cuanto a la disponibilidad de recursos, sino también en tiempos y, sobre todo, en la calidad y riqueza de experiencias, vivencias y oportunidades educativas que condicionarán directamente la adquisición de la CD.

\section{Contexto de la investigación}

Competencia digital e inclusión educativa. Visiones de profesorado, alumnado y familias. $\mathrm{M}^{\mathrm{a}}$ Montserrat 
Para hacer posible la integración de las tecnologías en las escuelas, las políticas se han acompañado de grandes esfuerzos por crear planes formativos para docentes en activo que permitan el desarrollo de su CD. También, en conseguir aumentar la dotación tecnológica y mejora de las infraestructuras para los centros (INTEF, 2017). Además, se han desarrollado medidas para la superación de las dificultades encontradas en los primeros tiempos como, por ejemplo, la conectividad, siendo mayores los obstáculos en zonas rurales de todo el territorio nacional.

En este marco, algunos diferencian entre los conocidos como nativos digitales (Prensky, 2001) y los emigrantes digitales (Area, Sanabria \& Santana, 2018). El término nativo digital se emplea para referirse al alumnado que ha crecido en un entorno tecnológico (Reyero, 2019). En cambio, el término emigrante digital se emplea para referirse al profesorado en activo que no necesariamente ha nacido en un entorno tan tecnológico como los jóvenes, no recogiendo la realidad diversa que se observa en las aulas.

Existe gran cantidad de estudios recientes centrados en cuestiones como la CD del profesorado (Fuentes, López \& Pozo, 2019), el impacto de las políticas educativas TIC en las diferentes etapas educativas (González Pérez, 2010), el grado de integración de las tecnologías en la escuela, los beneficios de la tecnología, las características de los materiales didácticos digitales (Castro, Rodríguez \& Peirats, 2017) o de los recursos educativos abiertos (Marjories, Campaña \& Gallego, 2018), el uso de recursos didácticos digitales frente a los tradicionales (Losada, Correa \& Fernández, 2017) y el análisis de portales de las administraciones educativas (González, Martín \& Vega, 2018; Santana, Eirín \& Marín, 2017). En muchos de los estudios recientes se tienen en cuenta las visiones de los profesionales y las familias, sin embargo, en pocos casos se tiene en cuenta la voz del alumnado, especialmente en la etapa de Educación Primaria. Excepciones son los trabajos de Martínez-Serrano (2019) y el de Rego \& Marín (2019) en el ámbito nacional, o los trabajos de Umar \& Jalil (2012) y Zhang \& Zhu (2016) en el internacional.

A pesar del interés creciente y los grandes esfuerzos que realiza la Administración educativa y los centros educativos, múltiples estudios encuentran una gran divergencia entre las políticas impulsadas por las Administraciones y las prácticas docentes en las aulas (Valverde \& Sosa, 2015). La metodología y los recursos utilizados, en demasiadas ocasiones, no varían respecto a épocas anteriores, a excepción del cambio de soporte al formato digital. Esta distancia entre las políticas y las prácticas puede deberse, entre otros factores, al nivel real de Competencia Digital Docente (CDD).

El trabajo que aquí se presenta forma parte de un proyecto de $\mathrm{I}+\mathrm{D}+\mathrm{I}$ en el que están implicados tres grupos de investigación: EDULLAB (Canarias), STELLAE (Galicia) y CRIE (Valencia). Este proyecto se organiza en cuatro estudios, siendo en el estudio III del proyecto de investigación en el que se pretendió identificar las representaciones o visiones que los agentes educativos tienen sobre el potencial didáctico de los recursos didácticos digitales en la enseñanza Primaria (Area, 2017). El proyecto se diseñó para ser desarrollado a lo largo de cuatro cursos escolares, siendo el tercer año, el momento en que se realizaron las entrevistas a los diferentes agentes educativos.

Los objetivos de este trabajo se centraron en:

1. Describir el grado de integración de las tecnologías en el ámbito organizativo y pedagógico de cuatro centros escolares. 
2. Analizar las visiones del alumnado, familias y profesorado sobre el uso académico de las tecnologías y sobre el desarrollo de la competencia digital.

3. Comparar las visiones de los agentes educativos para identificar los puntos de encuentro.

4. Valorar la inclusión del alumnado con necesidades específicas de apoyo educativo en las aulas de quinto y sexto de primaria de los centros participantes.

En este artículo, se describe la realidad de cuatro centros educativos de Educación Infantil y Primaria, ubicados en dos comunidades autónomas distanciadas dentro del estado español. El trabajo se centra en la utilización de tecnologías en los procesos de enseñanzaaprendizaje, pero desde la perspectiva de los verdaderos protagonistas de este proceso, los alumnos, sin olvidar en ningún caso la voz de las familias.

\section{Metodología}

Para alcanzar los objetivos señalados, la investigación se planteó desde un enfoque cualitativo, descriptivo-explicativo, que se caracteriza por permitir el reconocimiento y análisis de las perspectivas de los participantes contemplando la diversidad (Flick, 2018). Esto ha hecho posible comprender, desde la cercanía y de forma reflexiva, los procesos y fenómenos que se producen en el contexto específico de varios centros educativos.

El método de investigación elegido, el estudio de caso, permite investigar lo particular y único de una determinada realidad. Desde esta posición "el estudio de casos es una oportunidad de ver lo que otros no han visto aún" (Stake, 1998, p.115). Este método de investigación nos permite, gracias a su planteamiento holístico, identificar aspectos donde lo global se refleja en lo local.

En este marco metodológico, se realizó un estudio comparativo de casos (Bogdan \& Biklen, 1982; Lloyd-Jones, 2003; Merriam, 1988; Yin, 2003), lo que permitió analizar y comparar, similitudes y diferencias entre los centros participantes en el proyecto. Se estudiaron las trayectorias y proyectos implementados para el desarrollo de la CD en el contexto de implementación tecnológica favorecido por la Administración. Así como, las visiones de los diferentes agentes sobre los procesos y resultados obtenidos, estableciendo relaciones entre el uso de lo digital en las aulas y los aspectos relacionados con el proceso de enseñanza-aprendizaje que se ven alterados.

\subsection{Instrumentos}

Con el fin de dar respuesta a los objetivos planteados, el proceso de recogida de información consistió en la realización de entrevistas semiestructuradas a familias, alumnado y profesorado de cada centro. Las entrevistas facilitan acceder al mundo vivido de los sujetos, los cuales describen, utilizando sus propias palabras, sus experiencias, creencias y opiniones (Kvale, 2011).

Las preguntas de las entrevistas se construyeron desde las cuestiones de investigación planteadas en el proyecto y en relación a las categorías de análisis propuestas: valoración de los materiales didácticos digitales, uso personal de dispositivos y servicios digitales, uso académico de los dispositivos, servicios y materiales, y percepción del grado de integración de las tecnologías. En este sentido, la formulación de las preguntas de las entrevistas viene fundamentada por el periodo previo de investigación en los contextos y los resultados que hasta el momento surgieron en el marco del proyecto de investigación.

Competencia digital e inclusión educativa. Visiones de profesorado, alumnado y familias. $\mathrm{M}^{\mathrm{a}}$ Montserrat 
En cuanto a las familias, se pretendió recabar sus opiniones sobre el uso que se hace de las TIC en las escuelas en las que han escolarizado a sus hijos, así como sobre sus grados de satisfacción y el conocimiento del Plan TIC de cada centro.

Una vez realizadas las entrevistas, se procedió a su transcripción y posterior elaboración de informes en los que se recogieron las evidencias.

Específicamente, en este artículo nos centramos en las contribuciones realizadas por alumnado, familias y profesorado en las entrevistas realizadas como parte del estudio de caso. Destacamos en este sentido, la especial relevancia que tienen los testimonios del alumnado.

\subsection{Contexto y participantes}

En este estudio participó alumnado de quinto y sexto curso de Educación Primaria, de cuatro centros educativos. Dos de los centros se encuentran en la Comunidad Autónoma de Galicia, mientras que los otros dos centros se sitúan en la Comunidad Valenciana. Los centros gallegos reflejan la realidad de un contexto educativo rural del interior de Galicia. En un caso, el centro escolar pertenece a un núcleo rural alejado de la ciudad, mientras que en el otro caso, se sitúa en una zona que funciona como lugar-dormitorio de una parte fundamental de la población que trabaja en Santiago de Compostela. Los centros valencianos muestran, por su parte, las particularidades de dos centros urbanos, uno de titularidad pública y otro privado concertado.

El acceso a los centros se realizó en el marco de trabajo del proyecto en el que se enmarca esta investigación. Los centros fueron elegidos para participar en esta investigación por su reconocida implicación en el desarrollo e implementación de las tecnologías en la escuela. Por tanto, la selección de la muestra fue a propósito, por conveniencia o deliberada (Cook \& Campbell, 1979). En cuanto a las familias, se pidió la colaboración del AMPA para la realización de las entrevistas. En cada uno de los centros se entrevistó al coordinador TIC y al tutor del grupo objeto de estudio.

Con la finalidad de contextualizar el estudio, a continuación, se describen brevemente los cuatro centros educativos en base a: su contexto institucional y social, el perfil del alumnado participante y el profesorado que conforma el Claustro.

Caso 1. El centro es de titularidad pública, forma parte de la red de centros plurilingües de la Comunidad Valenciana y cuenta con tres unidades de Educación Infantil y seis de Educación Primaria. Uno de los ejes de trabajo en esta escuela es el uso de las TIC y una metodología de trabajo por proyectos. Los profesionales de los cursos superiores crean parte del material didáctico centrándose en los intereses del alumnado y son implementados desde un enfoque multidisciplinar, ya que apuestan por un uso limitado y sostenible de los materiales impresos, especialmente los libros de texto. Este centro participó en el Programa Experimental para el fomento del uso de libros de texto en dispositivos electrónicos denominado "Tabletas en centros educativos". Posteriormente, el centro continuó su labor de inclusión de las tecnologías. Hoy en día, las TIC tienen una gran presencia en el centro, especialmente en quinto y sexto curso, donde se trabaja con tabletas como principal herramienta didáctica. La CD y la competencia comunicativa son los aspectos fundamentales que se desarrollan en este centro, desde un enfoque transversal.

Competencia digital e inclusión educativa. Visiones de profesorado, alumnado y familias. $\mathrm{M}^{\mathrm{a}}$ Montserrat 
Caso 2. La titularidad del centro es privada concertada. Se trata de un centro diocesano dependiente del Arzobispado de Valencia y forma parte de una red de 68 centros diocesanos bajo la Fundación San Vicente Mártir. En el curso escolar 2014-2015 el centro inició un proceso de integración pedagógica de las TIC mediante un proyecto denominado "Mochila Digital". Los avances en relación a la implantación de las TIC a nivel organizativo y pedagógico han sido rápidos, continuando hoy en día perfilando diferentes aspectos de los procesos de inclusión tecnológica. En este sentido, desde $5^{\circ}$ de primaria hasta $4^{\circ}$ de la ESO, el alumnado del centro emplea las tecnologías y metodologías activas de aprendizaje. Específicamente, emplean IPad para el trabajo diario, el cual se centra en el alumno, en sus necesidades académicas, así como en el uso saludable de los dispositivos digitales, tal y como queda recogido en los documentos del centro.

Caso 3. Una de las características de este centro público es la agrupación multigrado en algunos niveles educativos. El alumnado de quinto y sexto, participantes en esta investigación, comparte el aula la mayor parte del tiempo y en algunas ocasiones se desdobla el grupo. A partir del curso 2014-2015 se inició un ascenso exponencial en cuanto a su participación en proyectos de innovación educativa y su presencia en la red. En buena medida, la innovación educativa del centro está en relación con la participación en proyectos, premios e iniciativas gubernamentales orientados a la adquisición de CD. Han obtenido numerosos premios y, en la actualidad, están desarrollando el Proyecto Edixgal, que consiste en la implantación de libro de texto digital en primaria y secundaria, y numerosos proyectos, planes como el Plan Proxecta: Aprendo programando, Proyecto GENIOS, Proyecto Escornabots, Club de ciencias, Semana STEM y Cibermanallers y laboratorio tecnológico IrimonLab.

Caso 4. Este centro público escolariza alumnado procedente en su mayoría del medio rural donde se combina una actividad agrícola y ganadera con otros trabajos, principalmente en la ciudad de Santiago de Compostela en los sectores de la construcción y servicios. Este es un centro pionero en Galicia en la introducción de las TIC desde el año 2000. Si bien no es un centro que haga una gran proyección de sus experiencias, existe una concepción generalizada de su tendencia innovadora, no sólo atribuida a las tecnologías. Han participan en distintos proyectos como Proyecto Abalar, Proyecto Edixgal o PROA. También han creado una red de formación interna para el uso pedagógico de la tecnología para el profesorado y el alumnado para adquirir una óptima CD. Asimismo, elaboran y adaptan materiales que comparten tanto dentro como fuera del centro. Los tienen organizados en una Intranet educativa. El trabajo se organiza por proyectos y otras metodologías activas sin utilizar libros de texto.

A continuación, en la Tabla 1 se resumen los datos más relevantes del contexto y los participantes entrevistados.

Tabla 1.

Información del contexto y participantes.

\begin{tabular}{|c|c|c|c|c|}
\hline & Caso 1 & Caso 2 & Caso 3 & Caso 4 \\
\hline Contexto & Valencia, urbano & Valencia, urbano & $\begin{array}{l}\text { Galicia, rural } \\
\text { (interior) }\end{array}$ & $\begin{array}{c}\text { Galicia, rural } \\
\text { (lugar dormitorio) }\end{array}$ \\
\hline Titularidad & Público & Concertado & Público & Público \\
\hline
\end{tabular}

Competencia digital e inclusión educativa. Visiones de profesorado, alumnado y familias. $\mathrm{M}^{\mathrm{a}}$ Montserrat 
RED. Revista de Educación a Distancia. Núm. 61, Artíc. 06, 30-10-2019

DOI: http://dx.doi.org/10.6018/red/61/06

\begin{tabular}{|c|c|c|c|c|}
\hline Estudiantes & 221 & 666 & 137 & 389 \\
\hline Etapas & EI y EP & EI, EP y ESO & EI, EP y ESO & EI y EP \\
\hline $\begin{array}{c}\text { Unidades por } \\
\text { nivel }\end{array}$ & 1 & $\begin{array}{l}2 \text { en EI y EP } \\
4 \text { en la ESO }\end{array}$ & Aulas multigrado & 2 \\
\hline \multicolumn{5}{|c|}{ Alumnado entrevistado (EA) } \\
\hline Número & 8 & 4 & 16 & 4 \\
\hline Curso & $5^{\circ}$ & $6^{\circ}$ & $5^{\circ}$ y $6^{\circ}$ & $5^{\circ}$ y $6^{\circ}$ \\
\hline Sexo & $4 \mathrm{M} \mathrm{y} 4 \mathrm{~V}$ & $2 \mathrm{M}$ y $2 \mathrm{~V}$ & $5 \mathrm{M}$ y $11 \mathrm{~V}$ & $2 \mathrm{M}$ y $2 \mathrm{~V}$ \\
\hline Edad & 10-11 años & 11 años & 10-12 años & 10-12 años \\
\hline \multicolumn{5}{|c|}{ Representante AMPA (EAMPA) } \\
\hline Número & 6 & 1 & 1 & 1 \\
\hline Sexo & Mujeres & Mujer & Hombre & Hombre \\
\hline \multicolumn{5}{|c|}{ Profesorado entrevistado } \\
\hline $\begin{array}{l}\text { Coord. TIC } \\
\text { (ECTIC) }\end{array}$ & 1 & - & 1 & 1 \\
\hline Tutor/a (ET) & $5^{\circ}$ & $6^{\circ}$ & $5^{\circ}$ y $6^{\circ}$ (cotutoría) & $6^{\circ}$ \\
\hline Otros docentes & - & - & $\begin{array}{c}\text { Matemáticas } 5^{\circ} \text { y } \\
6^{\circ}\end{array}$ & Inglés \\
\hline
\end{tabular}

Fuente: Elaboración propia.

\subsection{Procedimiento}

Para el desarrollo de este estudio se diferenciaron cuatro fases. La primera de ellas, consistió en el establecimiento de los criterios de selección de los centros y en el contacto con los mismos para informarles de los objetivos del proyecto y conseguir su compromiso y participación. La segunda fase fue en la que se recogió la información a través de la realización de las entrevistas a los diferentes agentes educativos. La tercera fase, implicó el análisis de los datos y la elaboración del informe de caso. Por último, se informó a los centros de los resultados obtenidos y el informe fue autorizado por los miembros del equipo directivo o por el Claustro de profesores en su conjunto.

\subsection{Análisis de datos}

Teniendo en cuenta que la información se recogió a través de entrevistas en profundidad, la técnica de análisis de contenido se ajusta a las necesidades de la investigación. La codificación se realizó mediante unidades y segmentos de información significativas. La información extraída se incluye en matrices de doble entrada, siguiendo un modelo de codificación axial, donde una columna identifica a cada sujeto entrevistado y en las filas, a su vez distribuidas en dimensiones, categorías y subcategorías, se recogen las respuestas 
y reflexiones de cada participante. El análisis de la información se realiza inicialmente por un miembro de cada equipo de investigación, para posteriormente, proceder a un análisis conjunto de todos los miembros que constituyen el equipo. En este sentido, se emplea la triangulación de investigadores con el fin de garantizar la consistencia lógica de las propias aportaciones en relación a los objetivos planteados y su representación en la realidad de los contextos (Miles, Huberman \& Saldaña, 2014). Además, se introdujeron las entrevistas en la herramienta Atlas.ti para identificar los tópicos, los puntos de encuentro en cuanto a los temas tratados y la singularidad de cada caso. Para finalizar, se realizó un informe que se le presentó al centro educativo, para que el Claustro realizase las consideraciones oportunas y lo aprobaran.

\section{Resultados}

Para la organización de los resultados se toman como base los objetivos de este trabajo. Por ello, en primer lugar, se presenta la descripción del grado de integración de las tecnologías en el ámbito organizativo y pedagógico de los cuatro centros escolares mencionados. En segundo lugar, se hace referencia al análisis de las visiones del alumnado, las familias y el profesorado, tanto coordinadores TIC como tutores, sobre el uso académico de las tecnologías y el desarrollo de la CD. También se trata la valoración que estos agentes realizan sobre la introducción de las TIC en la escuela. Por último, se describe la valoración de la inclusión del alumnado con NEAE en las aulas de $5^{\circ}$ y $6^{\circ}$ de primaria de los centros participantes y las posibilidades que la tecnología ofrece.

\subsection{Presencia y usos de los dispositivos tecnológicos para el desarrollo de la competencia digital}

En una investigación cualitativa es necesario introducir información que ayude a contextualizar las opiniones y visiones de los sujetos que forman parte de la comunidad y que participan en el proyecto de indagación. Por este motivo, es oportuno hablar de los dispositivos tecnológicos con los que trabaja el alumnado en la escuela en relación al grado en que estos están integrados en los procesos educativos y a nivel organizativo. Para ello, nos apoyamos en lo expuesto por De Pablos, Colás \& González (2010). Estos autores diferencian tres grados de integración de las TIC en el contexto educativo: introducción, aplicación e integración. El momento de introducción constituye el grado de integración de las TIC más bajo, pasando por la aplicación y siendo la propia integración el más alto. En cada uno de estos momentos cabe hacer referencia a las propias singularidades del proceso que se desarrolla en el contexto específico y en las dimensiones pedagógica y organizativa. En este sentido, son notorias las diferencias entre los cuatro centros analizados en las dos comunidades autónomas:

En el Caso 1, en relación al grado de integración de las TIC, respecto al ámbito organizativo, el centro continúa implementando el uso, mantenimiento y actualización de su web y blog, así como el uso de las TIC para fines administrativos y de gestión. Se encuentran en el periodo inicial de uso de las tecnologías para favorecer la comunicación e implicación de las familias en la política del centro, así como en su uso para facilitar la comunicación y coordinación entre los profesionales del centro. Respecto al ámbito pedagógico, se encuentran en periodo de aplicación de recursos digitales, así como de su producción como parte del proceso de enseñanza aprendizaje. Además, están en fase inicial del uso de las TIC en procesos educativos presenciales y virtuales. El uso de tecnología en el proceso de enseñanza-aprendizaje es una apuesta firme del equipo directivo y forma parte de la filosofía del centro. Sin embargo, el motor de la

Competencia digital e inclusión educativa. Visiones de profesorado, alumnado y familias. $\mathrm{M}^{\mathrm{a}}$ Montserrat Castro Rodríguez, Diana Marín Suelves y Héctor Saiz Fernández.

Página 12 de 37 
implementación de la tecnología en este centro se encuentra en los últimos cursos, quinto y sexto de primaria, siendo clave el papel de una de las tutoras que, además, es la encargada de la creación de los materiales didácticos digitales que se emplean en las áreas instrumentales en ambos cursos. En cuanto a los dispositivos disponibles en el centro destaca que se cuenta con 1 servidor, 16 clientes semi-ligeros de conexión a la red y 10 ordenadores multimedia, 1 proyector, 1 cañón, 1 escáner y 2 impresoras. Además, el alumnado de quinto y sexto trabajan con tabletas y no emplean libros de texto en papel.

En el Caso 2, en relación al grado de integración de las TIC, se encuentran en la etapa de aplicación a nivel organizativo respecto a su presencia en redes e internet, siendo reducida su actividad a la hora de actualizar los contenidos. En relación al uso de las tecnologías por las familias y los profesionales como medio de facilitar y favorecer la comunicación, en el centro existen plataformas y canales de comunicación digitales que han posibilitado la comunicación constante y bidireccional entre los miembros de la comunidad educativa. Además, el centro ha integrado las TIC y las emplea como medio para realizar tareas de administración y gestión. Respecto a la dimensión pedagógica, cabe señalar que el centro no cuenta con un plan específico de uso de las tecnologías. Sin embargo, tanto docentes como estudiantes emplean las tecnologías de forma activa y colaborativa en su labor académica y educativa. Se encuentran, al igual que a nivel organizativo, en el momento de aplicación. Por último, los materiales son, en ocasiones, creados por los profesionales del centro, siendo común que se trabaje con libros digitales de diferentes editoriales comerciales. Destaca el uso del iPad y la PDI, así como Google, Youtube (Happy learning), Popplet, Inspiration Maps, Apple TV, iTunes U o material escaneado y diapositivas. Están comprometidos con la integración de la tecnología en varios cursos y etapas educativas.

En el Caso 3, la comunicación a través de recursos digitales es el cauce más habitual para el intercambio de información con las familias. Tanto las familias como el profesorado vinculan las TIC con los procesos de innovación, considerándolas herramientas clave en el proceso de enseñanza-aprendizaje junto con otros recursos. En el discurso compartido se deja patente el rol fundamental que le atribuyen a los recursos y materiales tecnológicos respecto a su contribución para la proyección de un centro educativo en el contexto nacional e internacional. A nivel organizativo se encuentran transitando entre el momento de aplicación al de integración. Existe una idea permanente en el discurso: un colegio situado en un entorno rural gallego puede ser un referente de innovación donde las TIC juegan un rol fundamental. Su web, Facebook y blogs proyectan el día a día de sus aulas con el objetivo de abrirse a la comunidad. Se hacen eco de la intensa presencia del centro en los medios de comunicación debido a la adjudicación de proyectos, su participación en jornadas y congresos, especialmente el equipo directivo, de orientación e incluso sus estudiantes, así como por los premios obtenidos, entre otros reconocimientos. Buena parte de las noticias o presencia en las redes están relacionadas con la consecución de proyectos y premios relacionados con las TIC. Se detecta, no obstante, un desigual interés del profesorado por participar en los diferentes proyectos y en el uso de las TIC en las aulas, así como diversidad de interpretaciones a la hora de valorar pedagógicamente estos recursos. En este sentido, a nivel pedagógico se encuentran entre la introducción y la aplicación. Se observa una alta participación en actividades de formación y una alta producción de materiales digitales, aunque existen aulas donde el uso del ordenador y del libro de texto digital es lo hegemónico. Destaca la diversidad de dispositivos representativa de una actualización permanente respecto al mercado tecnológico: ordenadores portátiles y de mesa, impresoras (también 3D), escáneres, proyectores, 
pantallas digitales interactivas, fotocopiadoras, tabletas, kits de creación interactiva, kits de robótica educativa, cámaras de fotografía y vídeo, grabadoras de audio con micrófono externo, equipos de radio y un aula Croma. Se percibe una disociación en el tipo de materiales que utilizan en sus dispositivos digitales. En algunas clases se aprecia un uso mayoritario de materiales proporcionados por editoriales o colectivos externos, sin embargo, en los proyectos más específicos aparece el alumnado como productor de materiales.

En el Caso 4, las TIC están presentes entre los medios de comunicación con la comunidad. El uso de las TIC tiene una larga trayectoria en el centro. Existe una importante dotación de recursos en todas las aulas y niveles educativos: ordenadores portátiles y de mesa, proyectores LCD, pizarras interactivas, escáneres, cámaras de fotos digitales, cámara de vídeo digital, impresoras, etc. A pesar de que no constituye una prioridad la actualización permanente de recursos, sí se busca la participación en proyectos específicos que contribuyan a la adquisición de nuevos dispositivos. En general, existe un consenso respecto a un proyecto de centro que concibe las tecnologías como instrumentos importantes en la escuela por su valor pedagógico a un nivel igual al resto de muchos otros materiales y recursos que se utilizan. Se busca la adecuación de estos recursos a las demandas de las distintas metodologías que se utilizan en las aulas. No se mantiene un discurso de supremacía de estos materiales respecto a otros, sino que hacen más hincapié en cómo utilizarlos para que se adapten a las exigencias metodológicas desarrolladas para la consecución de los objetivos planteados. Existe diferentes posibilidades formativas que surge desde el propio centro, siendo principalmente autodidacta y cooperativa entre la mayoría del claustro. Respecto a las observaciones de aula, es desigual el uso integrado de recursos. A nivel pedagógico el centro se encuentra entre la introducción y la aplicación. Mientras que en unas aulas se prioriza el uso del ordenador para acceder al libro de texto digital, en otras, se opta por un mayor grado de complementariedad de recursos. En general, se opta por el uso de materiales adaptados y elaborados por agentes externos al centro que comparten en la intranet.

En resumen, a partir de la información ofrecida por los diferentes agentes educativos es posible identificar el grado de integración de las tecnologías, en cada uno de los centros analizados, en cuanto a las dimensiones pedagógica y organizativa, diferenciando tres posibles niveles: introducción, aplicación e integración. Tal y como se muestra en la Figura 1, en general, existen diferencias importantes entre los cuatro centros estudiados. Estos se encuentran en fases distintas de implantación tecnológica a pesar de que todos ellos cuentan con profesorado con capacitación en el uso de dispositivos tecnológicos. Además, la tecnología no solo está presente en el contexto del aula, sino que constituye un canal de comunicación activo con las familias. 


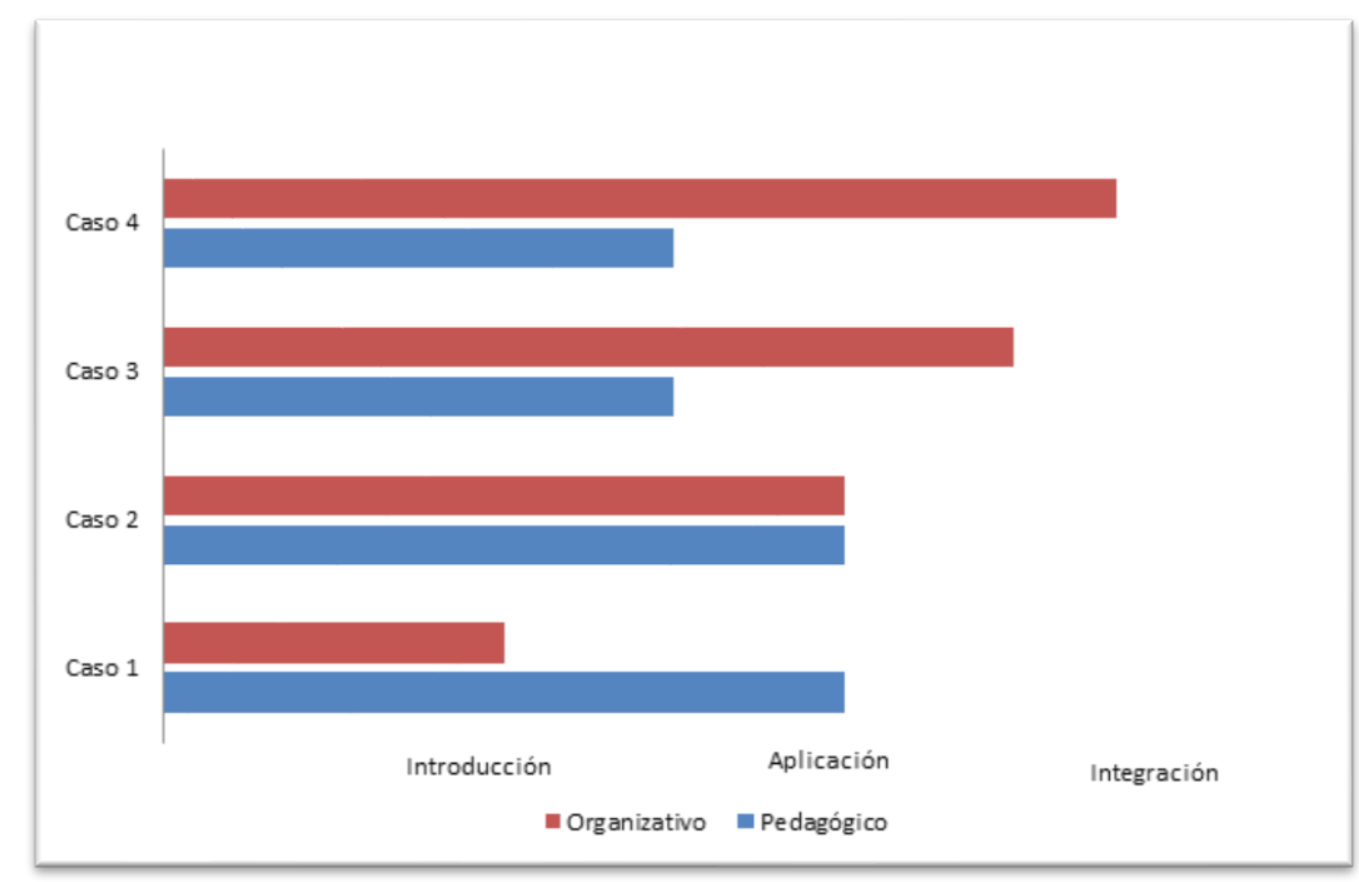

Figura 1. Grado de integración de tecnología en la escuela. Elaboración propia.

Cabe señalar también que, en cuanto a los dispositivos utilizados en los centros educativos, los soportes más comunes que se utilizan para el trabajo en el aula son los ordenadores, las PDI y las tabletas. La forma más habitual de trabajo con estos es mediante el uso de aplicaciones (app). Estos soportes utilizan diversas aplicaciones en base a los contenidos a trabajar, las materias académicas y los objetivos planteados en las tareas. En cada centro se utiliza un tipo de tableta, generalmente, la diferencia se centra entre los dispositivos con sistema operativo iOS (iPads) y con Android. En los centros públicos en los cuales se emplean tabletas, destaca el uso de aplicaciones de Google, ya sea para el trabajo académico, así como para la comunicación entre los miembros de la comunidad educativa. En cambio, en el centro privado concertado se emplean aplicaciones del servicio de Apple (como iTunes o AppleTV).

\subsection{Introducción de tecnología en el aula y competencia digital}

\subsubsection{Las visiones del profesorado}

Desde el punto de vista del profesorado participante en este estudio, la introducción y manejo de las TIC implican un modelo de enseñar y aprender en el siglo XXI centrado en las competencias necesarias para la vida. Para la construcción e implementación de este modelo, la CDD es considerada por los propios profesionales de la educación como la clave fundamental para llevar a la práctica el cambio metodológico, la innovación, que garantice la introducción de recursos y dispositivos tecnológicos como herramientas facilitadoras del proceso de enseñanza-aprendizaje. Tal y como afirma una de las docentes entrevistadas, valora positivamente la introducción y uso de estos dispositivos "por la variedad que tienes de hacer cosas, por la cantidad, por lo atractivo que lo puedes hacer para ellos, por lo motivador que les resulta" $\left(\mathrm{ET}^{\circ}{ }^{\circ} \mathrm{C} 2\right)$. 
Algún docente considera que la integración de las TIC no es garantía suficiente, ya que no siempre contribuye a una mejor adquisición de contenidos de materias concretas o de la propia CD. Así se refleja en esta afirmación: “...los conocimientos también requieren esfuerzo personal, manual, la comprensión lectora, una correcta estructura, una competencia matemática práctica para poder estar en la vida diaria. Entonces ahí a lo mejor pues hombre... una buena explicación, unos buenos ejemplos prácticos, la vivencia es muy importante" (EPC3). En contraposición, hay quien considera que las habilidades y destrezas relacionadas con la CD aportan al proceso de enseñanza-aprendizaje nuevas posibilidades para la adquisición de otras competencias desde una perspectiva innovadora, no sólo en las formas, sino que la introducción de otros contenidos inherentes al mundo tecnológico puede contribuir a un desarrollo más profundo de todo el alumnado: "En matemáticas lo que he descubierto es que es muy bueno trabajar el pensamiento computacional(...) es muy bueno trabajar con ellos la programación porque permite crear o generar estrategias de razonamiento lógico(...) mola mucho en lengua(...) para trabajar la oralidad es la radio que es una tecnología tradicional que con un uso emergente (...) va a permitir al alumnado que tenga una competencia oral muchísimo mejor (...) miedo a expresarse, a hablar y se trabaja cooperativamente, muchísimo mejor” (EPC3).

Un elemento de especial relevancia para una docente gallega es sin duda la contribución de la CD del alumnado a la superación de estereotipos, de barreras culturales y axiológicas que afectan, que conllevan la discriminación derivada de la brecha de género y de contexto social. Como refleja la siguiente cita: “...estamos en el medio rural, aquí (...) predominan las carreras de letras, las científicas no las tenemos. (...) Sobre todo para las chicas (...). Aquí es una sociedad aún bastante patriarcal. Tienen bastantes estereotipos (...) sociales, así bien marcados, y tienen que comprender nuestras chicas, nuestras rapazas que pueden hacer lo que quieran y que tienen que estar dentro de sus posibilidades las vías científicas y tecnológicas..." (EPC3). En este contexto se entiende la CD desde una perspectiva amplia, pues la alfabetización digital contribuye a acceder a la información, al mismo tiempo que se convierte en una oportunidad para comunicarse, intercambiar y crear conocimiento conjuntamente. Incluso puede favorecer la transformación social.

Los distintos discursos existentes en torno al uso de las TIC en el proceso de enseñanzaaprendizaje evidencian que la concepción del docente sobre sus competencias profesionales, experiencias y formación específica relacionadas con la $\mathrm{CD}$ y con el conocimiento y uso de las metodologías, podrán condicionar de forma importante el aprovechamiento de todas las herramientas y materiales digitales para que su alumnado adquiera una $\mathrm{CD}$ más completa e integrada.

En muchas de las explicaciones del profesorado, se aprecia la necesidad de que el alumnado adquiera CD para hacer frente a los retos del futuro. En este sentido, el desarrollo de la alfabetización digital y la capacidad de seleccionar la información en relación a su adecuación y veracidad es un punto clave dentro de las carencias que observan los docentes y de las habilidades a desarrollar como parte de la CD.

Respecto a la asunción de la función de coordinador TIC, en ocasiones, dentro de los Claustros el proceso no es sencillo, ya que como afirma el coordinador TIC del caso 1, en su momento la suya fue "por eliminación (...) tienes que tener ganas, que te guste e implicarte en el tema de gestión y que después tienes que tener ciertos conocimientos, pues seguramente hay tres personas que puedan tener ese perfil” (ECTICC1). Esto indica 
que gran parte del profesorado está en proceso de desarrollo de esta competencia y que entran en juego otras cuestiones como el tiempo, la sobrecarga de tareas, la necesidad de formación continua y la conciliación familiar. Para superar estas dificultades o barreras, en el caso 1, proponen la formación entre iguales, entre todo el profesorado del Claustro, ya que, en definitiva, la formación para hacer un uso pedagógico de las TIC depende de cada docente y su interés por la tecnología y la enseñanza. Reconocen que "existe una conciencia general en utilizar las TIC y formarse por todo el centro" (ECTICC1). Para ello, aprovechan las modalidades formativas que se ofertan desde los centros de formación al profesorado, en la Comunidad Valenciana desde el CEFIRE y en Galicia desde el CAFI. Esto se traduce habitualmente en la realización de "un seminario de formación en tabletas y, dentro del Plan Anual de Formación, se plantea alguna actividad que tiene que ver con las TICs" (ECTICC1).

En este mismo sentido, existen distintos niveles de formación entre el profesorado intra e intercentros. Tal y como afirma una maestra, para la elaboración de los materiales didácticos digitales es importante y necesaria la Formación Permanente del Profesorado, con el fin de poder implementar los recursos didácticos digitales en un centro educativo. Sin embargo, en su caso particular "esa formación ha sido muy autodidacta y con muchas horas" $\left(\mathrm{ET} 5^{\circ} \mathrm{C} 1\right)$, destacando la realización de cursos en el CEFIRE, como un curso sobre la página web de Lliurex, o centrados en el tratamiento de la imagen como Scratch. Respecto a las tabletas, esta misma profesional recibió "cursos de formación on line con la UOC de Barcelona porque aquí cuando se instauró el Programa de las Tabletas no había ningún curso" (ET5 $\left.{ }^{\circ} \mathrm{C} 1\right)$. Posiblemente, esta formación a veces deficitaria, a veces coyuntural, pueda explicar la visión restrictiva que en ocasiones manifiesta el profesorado de lo que entiende por CD.

El tener un buen nivel de desarrollo de la CD permite la introducción de materiales y recursos diferentes al libro de texto, ya que corresponde a cada docente la elección del material, el formato, soporte y la metodología de trabajo para cada clase. "Evidentemente no se trata de escoger, si no de saber qué recurso necesitamos para cada tipo de actividad o para cada momento" (EPC4). Además, la autoeficacia, percibida como el éxito que se obtiene al realizar una tarea, repercute en la satisfacción con el propio ejercicio profesional una vez se decide introducir el uso de recursos didácticos digitales. Entendiendo su carácter clave, sin embargo, consideran la necesidad de introducir el factor de complementariedad e incluso en algunos casos subsidiario de otros materiales. "No podemos desterrar el papel, (es necesario para) el trabajo, la concentración... sobre todo creo que los materiales físicos, en papel, sirven (...) para reforzar los conocimientos, para trabajarlos (...) las aplicaciones didácticas interactivas, otros recursos multimedia, los objetos digitales de aprendizaje (...) para consolidar los conocimientos y para que el alumnado haga su propia autoevaluación" (EPC3). Este discurso lo realiza un docente que hace un uso mayoritario del libro de texto digital, por tanto, parece que predomina un modelo híbrido en cuanto a la selección y uso de materiales didácticos.

La seguridad en el uso de la tecnología aparece en el discurso de los diferentes agentes, pero no se integra en el proceso de enseñanza-aprendizaje. Hablan de la necesidad del uso responsable, que se materializa muchas veces en las aulas observadas en la reflexión y explicación de medidas de seguridad, en algunos materiales producidos por estudiantes, el abordaje de temas como el ciberbullying o sexting y en la oferta de cursos de formación. 


\subsubsection{Las visiones del alumnado}

La mayoría de alumnado entrevistado en los cuatro centros muestran su agrado en relación con el trabajo con materiales didácticos digitales en el aula. Son varios los motivos que argumentan para justificarlo, aunque el factor motivacional intrínseco es el más compartido entre la mayoría del alumnado. Como afirma un estudiante valenciano "noto que aprendo más con la tablet porque tengo más ganas de trabajar que con un libro de texto" (EAC1.5).

Otra de las razones que exponen es su utilidad para la adquisición de aprendizajes escolares. En muchos casos, valoran positivamente el carácter hipertextual que puede ofrecerles un documento o material digital. Herramientas como aplicaciones, webs y blogs les aportan alternativas que complementan el estudio y el trabajo en el aula. Estas proporcionan actividades de aprendizaje y juegos que les facilitan la ampliación de los contenidos, aclaraciones e incluso les pueden ayudar a autoevaluarse: "no son solo juegos, también hay algunos, como en el libro de matemáticas, tú le pulsas y tiene como aclaraciones que a lo mejor en la teoría no están puestas, y es como otra cosa que tú deberías saber. Entonces tú le das al botoncito y te lo explica bien y todo" (EAC2.3). Para este estudiante "nos ayudan también a entender más las cosas... te ayudan a estudiar..." (EAC2.3) y como expresa otro, "te aclara las cosas" (EAC2.2).

Incluso cuando los contenidos son los mismos en formato papel y electrónico, los estudiantes manifiestan que lo digital les facilita su comprensión. Pero es necesario subrayar el discurso contradictorio del alumnado. En este contexto resulta significativo escuchar los relatos de los distintos estudiantes porque identifican qué es lo que perciben que les interesa a sus docentes que aprendan. Por ejemplo, en el centro 3, la mayoría del alumnado coincide en identificar el uso del ordenador e internet con la adquisición de contenidos conceptuales, bien para buscar información en el libro de texto o para realizar actividades en distintas webs, cuyo objetivo es fortalecer los aprendizajes. La CD, en este caso, se trabaja en un marco pedagógico relacionado con la adquisición y refuerzo de contenidos, lo que tradicionalmente se relaciona con los conocimientos escolares que recogen los libros de texto.

Otros estudiantes consideran como ventajas que "a la hora de estar haciendo deberes si no sabes algo puedes mirar las cosas... en Google o en cualquier otra plataforma informativa" (EAC2.3). Claramente, se identifica el potencial de las herramientas digitales como ayuda y apoyo a través de la búsqueda, que conlleva la autonomía y gestión propia de cada estudiante. Cierto alumnado argumenta que para estudiar, refiriéndose a la acción de memorizar, prefieren formatos tradicionales, frente a otros que rechazan los formatos tradicionales impresos $\mathrm{u}$ otros materiales de carácter manipulativo. En este sentido, en los colegios gallegos, reconocen el valor de la utilización de tecnologías en proyectos, basados en metodologías activas, desarrollando experiencias en la comunidad como proyectos solidarios, exposiciones, investigaciones, lo que facilita la proyección social al mismo tiempo que generan nuevos productos y adquieren nuevos aprendizajes que van más allá de los propios recursos tecnológico. Por tanto, se puede identificar la integración de varias dimensiones de la $\mathrm{CD}$, que supera la alfabetización, como la comunicación o las posibilidades para ser productores de contenidos. 
Otros de los aspectos positivos que argumentan hacen referencia al peso, la manejabilidad y la comodidad de transporte. Estas características aparecen frecuentemente en la mayoría de entrevistas.

La CD se asocia también a la ludificación del proceso de aprendizaje y en relación a las posibilidades de usar distintos tipos de dispositivos digitales para trabajar. En las conversaciones y observaciones de aula, se puede comprobar la fuerte implicación de la mayoría del alumnado en el uso de las TIC cuando se trabaja de forma lúdica. El discurso mayoritario surge en torno a lo atractivo que supone para ellos ciertos recursos y materiales más bien relacionados con la dimensión lúdica. Conocen numerosos programas, webs y juegos que no están vinculados a las materias.

Algunos estudiantes atribuyen a las TIC un valor muy positivo para establecer la comunicación y la interacción entre estudiantes y profesionales del centro, lo que se ve reforzado por la posibilidad de comunicarse a través de los dispositivos. A este respecto, expresan que "puedes preguntarle dudas... Entonces Eva, la profesora, se mete y te responde" (EAC1.4). Esto facilita el trabajo al alumnado desde el hogar cuando surgen dudas sobre las tareas. Consideran también que favorece el trabajo en grupo cuando un miembro del mismo no asiste al centro, por ejemplo, por enfermedad. Sólo tienen que "enviar fácilmente un Gmail, entonces ella se lo puede preparar y organizar" (EAC1.3) para que no se pierda el ritmo de trabajo. Sin embargo, otros estudiantes inciden en los efectos negativos para la interacción dentro del aula, argumentando que suelen ser causa de distracciones.

En general, el alumnado valora positivamente su CD sintiéndose seguro respecto a los los conocimientos derivados de su uso, el manejo de recursos y materiales y especialmente, en relación a saber jugar, buscar webs y desenvolverse en el proceso.

Las escuelas observadas destacan por la diversidad de recursos y materiales digitales que ponen a disposición del alumnado en general. Tanto estudiantes como profesorado de forma espontánea se refieren a buscadores, a programas, apps, webs, dispositivos digitales que utilizan en las distintas clases con bastante asiduidad. Son comunes a todos los centros estudiados los ordenadores, las tabletas, las PDI, impresoras y escáneres que manipula el propio estudiantado. Los alumnos, de forma general, diferencian entre aplicaciones "para aprender" o "para hacer documentos, tipo actividades o presentaciones" (EAC2.2).

Respecto al uso cotidiano de las TIC en los centros, sus búsquedas habituales se realizan en Google (así como en las plataformas asociadas), Diccionarios, Wikipedia y WordReference. Los programas más utilizados para realizar sus trabajos son LibreOffice, Wifi, Anki, LibreOffice Impress, Google Drive, Scratch, Documents, BookCreator, los blogs, Webquest, Pasapalabras, Kahoot, Edilim, eXeLearning. Destaca también el uso de la robótica. En las clases de inglés, adquieren protagonismo mBlock, English Blog, QuickSIS, Duolingo, Learn by English y diferentes diccionarios. Existen, en este sentido, características diferenciales en función de la comunidad autónoma. Los colegios gallegos usan los libros de texto digitales que les proporciona el proyecto Edixgal de la Consellería de Educación. En los colegios valencianos, destacan las aplicaciones que les permiten acceder a libros en formato digital, como Savia e iTunes, para exportar o trabajar de forma independiente los contenidos de los libros. También destacan en el contexto valenciano las apps que se emplean para la elaboración de mapas conceptuales (InspirationMaps, Popplet) que favorecen su capacidad de atención. Como expresa un estudiante "me ayuda 
a centrarme, porque es todo el tema y dividido por partes, como un mapa mental" (EAC2.1). También otras para el desarrollo de competencias matemáticas y de resolución de problemas (Arloon Mental Maths), relacionadas con competencias sociales y culturales (Google Earth) y con competencias lingüísticas (EduDigital), entre otras.

El alumnado de estos centros se reconoce como nativo digital por la percepción de tener un amplio conocimiento sobre herramientas que utilizan en distintos contextos. Sin embargo, como muchos de ellos exponen, las experiencias educativas, los usos y aplicaciones de la CD en la escuela está muy condicionada por el profesorado. A lo largo de las entrevistas, en todos los centros, realizan comentarios sobre las distintas formas que tienen de trabajar con un docente u otro. En general, el alumnado percibe los distintos enfoques del profesorado, que deambulan entre prácticas tradicionales y las más innovadoras; perciben la continuidad o discontinuidad de la integración en el día a día, que oscila desde el uso exclusivo hasta la complementariedad con otros materiales no digitales. En muchas de las respuestas del alumnado se recoge el papel de guía o director de cada docente, que está orientado a una parte importante de experiencias dirigidas, planificadas y, en varios casos, condicionadas por el profesorado. Estas conviven con otras oportunidades, menos frecuentes, en las que el alumnado tiene que desarrollar toda la actividad de forma autónoma, participando de forma más libre y favoreciendo aspectos de la CD como su uso creativo, crítico y seguro.

En general, el alumnado adopta un sentido crítico a la hora de identificar los materiales más adecuados de acuerdo con sus intereses escolares y de ocio. Priorizan los materiales externos a los que crean los docentes, ya que les ofrecen más posibilidades de interacción y de ampliación de contenidos, principalmente por la diversidad de enlaces presentes en el documento. Respecto a la pregunta sobre por qué el resto de materiales, que no tienen que ver con el libro digital, no permiten ampliar información, el alumnado responde que "no porque los documentos los hacen las profes, no los hace el libro" (EAC2.1).

En algunas respuestas hacen referencia a los peligros del uso de las redes, sin embargo, no lo asocian directamente con la adquisición de $\mathrm{CD}$. Cuando se les pregunta si consideran necesario aprender a usar adecuadamente internet y las redes, responden afirmativamente $\mathrm{y}$ aluden a algunos de los peligros que tiene el uso de las redes. No obstante, no lo asocian a adquirir conocimientos que les puedan ser útiles en el contexto académico, ni como algo capaz de ser evaluado. Respecto a los riesgos, en las conversaciones surgen las voces de quienes consideran excesivas las horas que permanecen delante de las pantallas y a las repercusiones físicas que de esta situación se derivan.

Resulta muy interesante como el alumnado valora positivamente su $\mathrm{CD}$, lo que les proporciona autonomía a la hora de realizar prácticas tecnológicas en sus casas, como la construcción de robots que luego comparten con sus compañeros y compañeras en el aula. También cuando "muchos de los trabajos finales que se les piden son materiales digitales, porque se piden entrevistas, se piden vídeos y todos los trabajos escritos siempre van por digital, se usan mucho las aulas virtuales (...) y ahí crean ellos (EDC3). Es quizás, en estos escenarios, donde el alumnado asume el rol más autónomo, sin la dirección del docente, lo que le permite ser más creativo.

Mayoritariamente son conscientes de algunos de los peligros que tiene el uso de los recursos y materiales digitales, y a los que no siempre son capaces autónomamente de darles solución. Así, exponen que en ocasiones se encuentran "muy distraídos con la tablet" (EAC1.5). En una de las aulas observadas, el alumnado se mueve autónomamente 
por el aula en muchas sesiones y mantienen conversaciones entre sí que no tienen que ver con la práctica que se está desarrollando en el aula, sino que tratan sobre webs, apps y otros materiales lúdicos. Resultan ser también fuente de conflictos en la convivencia, con origen en las carencias de habilidades para la gestión de los conflictos, también en falta de responsabilidad en el uso.

Los alumnos viven con preocupación si cuando lleguen al instituto sabrán trabajar con otros recursos no digitales. Determinados estudiantes valoran más unos recursos que otros: "Primero sería mejor que aprendiesen en papel y luego... En quinto y sexto ya se hacen con el ordenador (...) Porque las tablets pueden 'viciar' y es muy malo..." (EAC4.3). Otro aspecto negativo que resalta es cuando realizan exámenes digitales, por los muchos problemas que esto acarrea.

\subsubsection{Las visiones de las familias}

Frente al alumnado, que en general aporta mucha información y valoraciones comunes, en relación a la perspectiva de las familias se aprecian diferencias significativas entre los cuatro centros donde se realizó el estudio. Pueden influir en estas diferencias los diferentes tiempos que los centros llevan integrando las TIC, así como la prioridad que le dan a este aspecto en el proyecto educativo de cada uno de ellos.

Existe un consenso entre los representantes de las AMPAS de todos los centros sobre la importancia del uso de las tecnologías como instrumentos de comunicación. Consideran que el uso de las TIC favorece la fluidez de la comunicación escuela-familia. En el análisis de las conversaciones, quizás haya una mejor comprensión de su importancia en la relación entre adultos, más que en la que se pueda dar con el alumnado. En los casos 1,2 y 3 destaca el empeño de los centros por habilitar canales y plataformas de comunicación con las familias. En algunas de las entrevistas, es patente que es más un deseo del centro, que una demanda de las familias.

En general, parece que en todos los centros está normalizado el uso de recursos digitales para la gestión y administración, tanto con el resto de la comunidad, como con las familias. En consecuencia, con la aceptación y normalización de las comunicaciones, las gestiones y la administración a través de las herramientas digitales, se aprecia una concienciación mayor sobre la necesidad de trabajar la CD.

En el Caso 1, las familias consideran que estas herramientas facilitan la coordinación entre los profesionales del centro, elemento clave para el buen funcionamiento del grupo clase. Sin embargo, no se aprecia un discurso tan unánime cuando se habla sobre el trabajo de la $\mathrm{CD}$ en el centro con el alumnado. En este caso, a pesar de que la digitalización del centro tiene una larga trayectoria, las familias no aportan mucha información sobre sus percepciones. Consideran que puede ser interesante y necesaria, pero se encuentran en situación de vigilancia y expectación, excepto en los casos en los que tienen hijos mayores que ya han realizado la transición a la etapa de secundaria, la cual suele ser exitosa gracias a los aprendizajes y competencias que reconocen haber desarrollado en este centro. En este caso, el impulso de las familias al proyecto de integración de las tecnologías ha sido determinante, ya que son ellos los que han asumido los costes de la compra de los dispositivos y de un wifi que permita el trabajo continuado de casi medio centenar de alumnos al mismo tiempo. Existe acuerdo al considerar que es necesario el uso de las TIC en las aulas para el desarrollo del proceso de enseñanza aprendizaje, valoran positivamente los recursos tecnológicos disponibles en el centro y el uso pedagógico que 
realiza el profesorado. También atribuyen y concuerdan en la necesidad de que el profesorado posea amplia CDD.

En el Caso 2, algunos de los materiales son creados por los profesionales del centro, sin embargo, lo más común es que se trabaje con libros digitales de diferentes editoriales comerciales. En este caso, se señala la importancia de la tecnología en la comunicación de las familias y en la gestión del centro y aula, tal y como afirma un representante del AMPA: "El cole tiene una plataforma que se llama Educamos que a través de ahí pues vemos notas, vemos incidencias, las excursiones, vemos todo. Lo del papel se quedó atrás. Además, te llega mejor por educamos, porque el papelito o bien te llegaba que no te llegaba. Así te enteras de todo, de autorizaciones, de todo. Entonces yo esa plataforma la veo muy bien" (EAMPAC2).

Tanto en el Caso 1 como en el Caso 2, cabe señalar que el centro no cuenta con un plan específico de uso de las tecnologías. Sin embargo, tanto docentes como estudiantes emplean las tecnologías de forma activa y colaborativa en su labor académica y educativa.

En el Caso 3, desde el AMPA se valora positivamente la implantación de las tecnologías. Su representante expone lo siguiente en relación a los comienzos del programa: "sobre este tema hubo cierta polémica (...) ¿Para qué quiere un niño de diez u once años un móvil? (...) no son sólo móviles: es una herramienta de trabajo" (EAMPAC3). Consideran a su alumnado como nativo digital con una alta $\mathrm{CD}$ con la que consiguen "romper un poco esa brecha digital" (EAMPAC3) en el ámbito rural. El alumnado usa herramientas digitales de máxima actualidad que le proyectan "también a la comarca, mostrando una actuación de puesta en valor de su configuración como pueblo y de los recursos institucionales" (ECTICC3). Sin embargo, "en sus hogares no pueden (tener acceso a la red), es una forma de que colaboren en igualdad de condiciones con sus compañeros/as" (EAMPAC3). Tal como expone un familiar de este centro "hay muchos padres que no se pueden permitir tener internet en casa (...) y así el niño/a va a disponer de ese servicio: no importa si tu padre tiene más o menos dinero, no importa si tus padres -porque podría haber algún caso- se preocupan o no por tu educación o le dan importancia a ese aspecto de tu educación... tú lo vas a tener" y se están preparando para el futuro "el día de mañana, puedes aprender a programar un brazo robótico y no tienes porqué cavar con la azada" (EAMPAC3). En general, las familias valoran negativamente el uso del libro de texto digital del programa E-DIXGAL, principalmente, por su escasa calidad y contribución a la adquisición de CD.

En el Caso 4, las familias consideran que las aportaciones de las TIC en algunas materias son mínimas y no contribuyen a mejorar el proceso de enseñanza y aprendizaje. Argumentan que la $\mathrm{CD}$ es una de las competencias a trabajar en el aula y quizás otros materiales contribuyan mejor a adquirir las otras. Valoran positivamente la accesibilidad a los recursos digitales que hay en el centro, así como la diversidad de experiencias que tiene el alumnado para aprender a utilizarlo. Encuentran carencias en el proceso de adquisición de la CD del alumnado en cuanto al uso de las TIC para fomentar la participación y la colaboración con el resto de la comunidad. No consideran que el uso que se le está dando a la página web del centro, blogs y uso de redes sociales se esté aprovechando para una mejor formación.

\subsection{Comparación de las visiones de los agentes educativos}

Competencia digital e inclusión educativa. Visiones de profesorado, alumnado y familias. $\mathrm{M}^{\mathrm{a}}$ Montserrat 
Del análisis de las entrevistas realizadas a familiares, alumnado y profesorado en los cuatro centros educativos emergieron los elementos que consideran relevantes para hacer posible la integración de la tecnología en la escuela y el desarrollo de la CD. Estos elementos surgen del análisis de contenido, de las manifestaciones que son compartidas por los diferentes agentes.

Para conseguir identificar los elementos clave en este proceso se partió de la identificación de las palabras más frecuentes en las entrevistas, utilizando como herramienta el programa Atlas.ti. Posteriormente, se procedió a la agrupación de estas palabras en grandes categorías, emergiendo un total de 5, tal y como se refleja en la Figura 2. Tanto las familias, como el alumnado y el profesorado destacan, en primer lugar, el valor de todos los agentes implicados para la construcción de una escuela digital que permita el desarrollo de la CD. En segundo lugar, señalan la importancia del diseño de los procesos de enseñanza-aprendizaje desde una perspectiva pedagógica clara encaminados a la consecución de la CD. En tercer lugar, poseen una mirada amplia sobre los contextos educativos, que van más allá del centro y el aula. En cuarto lugar, se explicitan las transformaciones que observan en los materiales didácticos, predominantemente digitalizados en los últimos tiempos. Y por último, destacan las metodologías activas implementadas, porque otras prácticas son posibles.

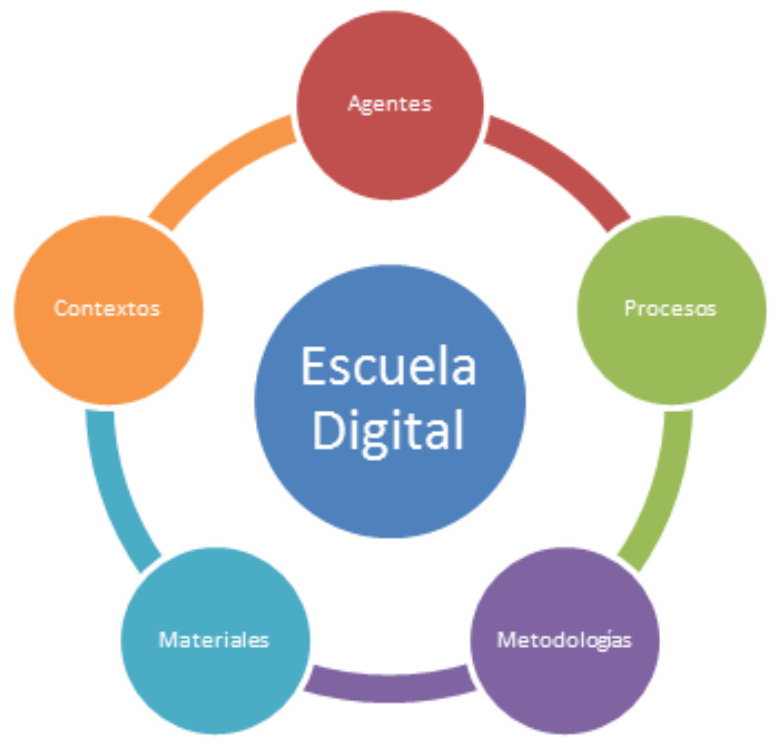

Figura 2. Elementos clave en la creación de la escuela digital. Elaboración propia.

\subsection{Valoración de la inclusión del alumnado con necesidades específicas de apoyo educativo (NEAE) en las aulas.}

En los cuatro casos analizados destaca la existencia de alumnado con NEAE, por Necesidades Educativas Especiales (NEE) derivadas de discapacidad intelectual o trastorno de espectro autista, o bien, por condiciones personales o historia familiar. Todos ellos escolarizados en su grupo ordinario de referencia, lo que supone el entorno menos restrictivo posible y la aplicación del principio de inclusión en el ámbito educativo.

Por ejemplo, en el caso 1 el alumnado con NEAE está escolarizado en un contexto inclusivo, en pocas ocasiones reciben el apoyo fuera del aula. Los grupos de alumnado van cambiando de miembros, para favorecer la socialización y el aprendizaje de todos. 
Esto permite la creación de un buen clima de trabajo y prepara para la vida en una sociedad diversa.

En general, el profesorado de las escuelas, no hace referencia a la adquisición de la CD del alumnado que presenta NEAE. Se visualiza una perspectiva de los materiales didácticos útiles para la accesibilidad al conocimiento, especialmente ligadas a áreas de conocimiento, aprendizajes específicos o motivacionales. Un miembro del Claustro que durante años ha encabezado el equipo directivo del Caso 1 afirma que "lo que le ayuda a ese alumnado, es que esa forma de trabajar lo hace más atractivo, más fácil, tiene más logros que fracasos" (EEDC1). En todos los casos se pone especial énfasis en la importancia de "facilitar el acceso a esta herramienta por parte del alumnado con necesidades especiales de apoyo educativo y en las tareas de apoyo y refuerzo de aprendizajes" (ECTICC3), dedicando un apartado concreto del documento del centro correspondiente a detallar los esfuerzos y medidas a favor de la atención a la diversidad, apostando decididamente por una educación integradora e inclusiva. Reconocen el valor de los materiales didácticos digitales por sus características pedagógicas, lo que les hace ser apropiados para todo el alumnado y especialmente para el que presenta NEAE por la cantidad de imágenes y por la motivación que generan. Como expresa un docente del centro 4, los materiales digitales permiten la personalización del aprendizaje, contextualización de la enseñanza, atención a la diversidad... posibilitan al profesorado "tomar decisiones que con los materiales tradicionales no podíamos, un libro de texto impreso está impreso y ahí no se puede tocar nada [...] El material digital ahora mismo nos está permitiendo adaptar posibilidades, incluso utilizar diferentes recursos para diferente tipo de alumnado dentro da propia aula" (EPC4) y “...gran ventaja de poder preparar material adaptado, en los dos últimos años tenía un niño Asperger, este año tengo un niño con dificultades de lenguaje y comprensión lectora importantes (tiene una ACI), poder crear materiales propios para ese niño, (...), no hay ninguna editorial que tenga este material es para los niños que tengan esta dificultad concreta" (EPC4). En este caso la CD está vinculada a la alfabetización del alumnado, posible creación de contenidos por parte del profesorado y solucionar problemas de accesibilidad.

En esta misma línea, el profesorado considera que la CD juega un rol fundamental en la socialización y contribuye a construir el conocimiento en el aula de Educación Primaria, ya que "las TIC facilitan situaciones de aprendizaje en las que se ha de dialogar, cooperar, etc. Y eso facilita situaciones adecuadas de interacción en el aula de Primaria. Sabido es que cuanto más interaccionen los alumnos más oportunidades tienen, más experimentan, más avanzan" $\left(\mathrm{ET} 5^{\circ} \mathrm{C} 1\right)$.

En algunos casos, en los relatos del alumnado de los centros analizados se hacen referencias concretas que podrían entenderse como medidas específicas de atención a la diversidad, concretadas en:

- la adecuación de algunas de las actividades que realizan a las necesidades de cada niño o niña.

- la apuesta por las actividades simultáneas en la misma clase que pueden entenderse como medidas de adaptación a los distintos ritmos y necesidades.

- algunos estudiantes comentan como las aplicaciones que usan o los accesorios facilitan la accesibilidad a contenidos, también a la realización de ciertas actividades. 
Las familias, en general, no relacionan las posibilidades que proporcionan las TIC con una mayor adaptación a las necesidades individuales. En algunas entrevistas, consideran que la adquisición de CD por todo el alumnado es una forma de promover la igualdad de oportunidades, pero entendiendo éstas para personas que se encuentran en contextos socioeconómicos y culturales más vulnerables, como ocurre en el caso 3 . En el centro 4, incluso consideran que el uso de las tecnologías no contribuye a la mejora de las experiencias educativas en algunas materias.

\section{Discusión}

En los últimos tiempos, uno de los grandes cambios que se ha introducido en la vida de personas de diferentes generaciones, etnias, procedencias y condiciones ha sido el impacto de la tecnología en todos los ámbitos (Peirats, Gallardo, San Martín \& Waliño, 2016). En este contexto, existe un acuerdo respecto a la conveniencia del abordaje del proceso de enseñanza-aprendizaje desde las competencias, entre las que destaca la competencia digital (Bender, Urrea \& Zapata-Ros, 2015), aunque surgen voces que, como ocurre en la presente investigación, cuestionan la edad del alumnado y la tipología de dispositivos utilizados, así como la naturaleza y contenidos de la CD (Juhaňák, Zounek, Záleská, Bárta \& Vlčková, 2019).

Con la realización de este estudio se pretendió conocer las visiones del alumnado, familias y profesorado sobre la integración de la tecnología en la escuela, sobre si el uso académico de ellas contribuye a la adquisición de CD. Además, nos centramos también en las implicaciones para la inclusión de todo el alumnado, así como en la valoración del uso de las TIC. Los trabajos en los que se considera la visión del alumnado son escasos (Sánchez, Alba \& Sánchez, 2018), al igual ocurre con aquellos que se refieren sólo a las familias (Martínez-Piñeiro, Vila Couñago \& Gewerc, 2018; Gamito, Aristizabal \& Vizcarra, 2019) o a ambos colectivos (Gewerc \& Martínez-Piñeiro, 2019). Para superar esta limitación de otros estudios, en este trabajo se contempla, además de las visiones del profesorado, las de otros agentes educativos específicos, las familias y el alumnado.

Se realizó un análisis comparativo de casos en cuatro centros singulares de dos comunidades autónomas del estado español. Para el análisis se tomaron como referencia las entrevistas realizadas con alumnado de quinto y sexto de Educación Primaria, representantes del AMPA y profesorado, especialista o generalista, y coordinador TIC de cada escuela.

En general, los resultados indican los grandes esfuerzos de la escuela por adecuarse a las características de la sociedad y del alumnado de este siglo. Hacer uso de la tecnología desde una perspectiva pedagógica conlleva beneficios para la mejora de la inclusión educativa (Pérez-Escoda \& Fernández-Villavicencio, 2016), en particular del alumnado con NEE (Marín, Morote \& Aguasanta, 2019). Además, generalmente supone una valoración positiva de alumnado, familias y profesorado en cuanto al desarrollo de la CD (Peirats, Gabaldón \& Marín, 2018).

En cuanto al grado de integración de las TIC en el sistema educativo, los datos indican que tomando como referencia los tres niveles descritos por De Pablos, Colás \& González (2010): introducción, aplicación e integración, en los cuatro casos analizados existe diversidad en el grado de integración de tecnologías en los procesos de enseñanzaaprendizaje y de gestión del centro. Esto viene a reflejar el impacto desigual de las políticas educativas aplicadas de forma descentralizada en cuanto a la tecnología en los 
centros educativos (Area, Hernández \& Sosa, 2016). Frente a lo expuesto por MartínezSerrano (2019), en los cuatros casos analizados se puede decir que existe una relación estrecha entre los recursos disponibles en las aulas y el uso de estas herramientas en el proceso de enseñanza-aprendizaje y en relación a la CD (Gutiérrez Martín \& Tyner, 2012). No se han identificado cambios sustanciales en las metodologías empleadas debido a la tecnología en sí. Sin embargo, cabe señalar que posibilitan la modificación del rol de los diferentes agentes educativos a través del uso de metodologías activas que emplean las TIC como, por ejemplo, los proyectos de trabajo.

En los cuatro centros, el alumnado vive en un ambiente donde se trabaja por la adquisición de la CD en el día a día. Observan cómo el profesorado y familias usan la tecnología para comunicarse y van superando las barreras de comunicación que surgen en el proceso (Bordalba \& Bochaca, 2018). Ellos mismos, a través de las distintas tareas y actividades que se realizan en el proceso de aprendizaje, entrenan distintas habilidades para establecer comunicación con la comunidad próxima y la sociedad en general. De las cuatro escuelas analizadas, una de las diferencias más evidente se encuentra en las distintas formas de trabajar la CD para la comunicación social. Se observa que un centro gallego vincula la adquisición de CD para la comunicación social a través de su proyección en las redes y en todos aquellos foros que le hacen visible como centro de innovación pedagógica, pudiendo superar así la brecha digital que implica estar situado en el contexto rural (Del Moral, Villalutre \& Neira, 2014; Gonçalves, Fernández, Castro, Ricoy, Rodríguez \& Cid Fernández, 2018). Estos incluso participan en proyectos de aprendizaje servicio con las TIC como una forma de transferencia del conocimiento. Se trabaja con el alumnado en la adquisición de conocimientos sobre cómo gestionar la información (formato, difusión, visibilidad) para que llegue a la ciudadanía, más allá de la propia comunidad. Perryman \& Jeng (2019) los identifican como líderes para el crecimiento de la comunidad. En otros centros, la CD para la comunicación en la sociedad se trabaja en la escuela, para la propia escuela y la comunidad escolar próxima. Destacan las diferencias encontradas en cuanto a la concepción y usos que se le atribuyen a las tecnologías dentro del proyecto educativo general y en particular reflejan el debate existente en la sociedad sobre la naturaleza y los contenidos de la CD (Pötzsch, 2016). En algunos casos, focalizan los procesos de innovación en relación al uso de la tecnología, frente a otros centros donde la innovación se vincula a metodologías (Spante, 2019) o incluso a contenidos y objetivos relacionados con el pensamiento computacional.

Respecto al uso de la tecnología, según el alumnado y sus familias, en todos los casos destaca el uso combinado de tecnología con recursos no digitales en mayor o menor grado. Por tanto, trabajan desde un modelo híbrido, en el que la convivencia entre lo analógico y lo digital es frecuente y consideran además que es lo más beneficioso. Esto ya se mostraba en estudios previos donde se concluye la pérdida de la hegemonía del libro de texto impreso sobre el digital (Sanabria, Álvarez \& Peirats, 2017). Tal y como explica el alumnado, es el profesorado el que selecciona el material o los recursos que va a utilizar, realizando también las adaptaciones para responder a las características de sus grupos-clase. En el Caso 1, los alumnos y familiares son conocedores del esfuerzo de las docentes al crear los materiales para cada asignatura, compartirlos y revisarlos cada curso escolar.

Para el profesorado entrevistado, la integración digital origina nuevos espacios de aprendizaje y experiencias (Van de Oudeweetering \& Voogt, 2018) que conllevan también el aprendizaje de muchos otros contenidos, más allá de los propios y específicos

Competencia digital e inclusión educativa. Visiones de profesorado, alumnado y familias. $\mathrm{M}^{\mathrm{a}}$ Montserrat 
de una materia. Por ejemplo, con respecto a las posibilidades de realizar multitasking o multitarea, el profesorado entrevistado también pone de manifiesto que con las TIC se pretende y se favorece que el alumnado sea capaz de mantener su atención en varias acciones al mismo tiempo, siendo para ellos en general algo positivo. Se puede decir, que, mayoritariamente en los centros analizados caminan entre las TIC y las TAC, sin poder hablar todavía de una integración total, con grandes diferencias entre las dimensiones organizativa y pedagógica. Sin embargo, mayoritariamente el profesorado reconoce su escasa CDD para la producción de medios, concordando con los resultados encontrados por Anggeraini, Faridi, Mujiyanto \& Bharati (2019), según los cuales un 77\% de la muestra de profesorado, tiene baja competencia para elaborar materiales propios.

La tipología de los dispositivos que puede utilizar el alumnado también es un aspecto relevante a la hora de trabajar la CD. En general, se constata la existencia de diversidad de recursos, de software innovadores, nuevas aplicaciones, incluso nuevos contenidos competenciales (De Almeida, Teixeira \& Almeida, 2019). Suele haber una hegemonía de uso por parte del alumnado en cada escuela, fundamentalmente ordenadores y tabletas, y con menos frecuencia las PDI, que se limitan al uso para la proyección y las presentaciones grupales. En los cuatro centros, la CD está mayoritariamente ligada a un modelo que identifica al alumnado como consumidores de tecnología (Hsin, Li \& Tsai, 2014). Desde el punto de vista de la manipulación de los recursos, las habilidades necesarias para el uso son diferentes en función de los dispositivos, así como del software y el uso que se puede hacer de los accesorios con los que cuentan unos y otros. En este sentido, estas distinciones no tendrían por qué contribuir a introducir desigualdades que pueden condicionar la adquisición de $\mathrm{CD}$, pero debería ser una cuestión a tener en cuenta en investigaciones futuras.

Cuando al alumnado se le pregunta directamente por los aprendizajes adquiridos al usar dispositivos digitales, en su mayoría, hacen referencia a estrategias o instrumentos que les facilitan el acceso a la información, así como al apoyo para el aprendizaje concreto de temas específicos vinculados a materias. En algunos de sus comentarios aluden al hecho de que los materiales que utilizan les proporcionan actividades o links que usan directamente. Es decir, el alumnado en muchas ocasiones, utiliza de forma directa los accesorios proporcionados por quien produce el material, pero en muy pocas ocasiones se visibiliza el trabajo para la adquisición de autonomía en la búsqueda de instrumentos o estrategias, aspecto esencial para una CD íntegra. Además, en general, todo el alumnado reconoce positivamente el potencial lúdico del uso de medios tecnológicos.

El profesorado identifica la adquisición de la $\mathrm{CD}$ del alumnado con la mejora del rendimiento académico, debido fundamentalmente a una mayor accesibilidad y cierta adaptación a cada sujeto, pero el factor motivacional adquiere un especial protagonismo, lo que viene a corroborar el estudio realizado por Del Moral, Villalutre \& Neira (2014).

Por otra parte, destaca el uso de la tecnología como herramienta que favorece los procesos de inclusión de todo el alumnado y, especialmente, del alumnado con NEE. Los docentes señalan que las TIC permiten la participación y aprendizaje de todos, por su carácter motivador y por las posibilidades que ofrecen para el ajuste a las características y ritmos de aprendizaje individuales, aunque no relacionan la adquisición de CD como un oportunidad para la mejora de las habilidades físicas y cognitivas como concluye la investigación de Ojeda-Castelo, Piedra-Fernandez, Iribarne \& Bernal-Bravo (2018) cuando el alumnado con diversidad funcional trabaja con el KiNEEt. Por tanto, la 
adquisición de $\mathrm{CD}$ de las personas con NEE, de acuerdo con las modalidades identificadas por Price-Dennis, Holmes \& Smith (2015), están reducidas a las adquiridas en prácticas escolares restringidas al uso de herramientas que les permiten acceder al currículum y a la adquisición de objetivos académicos, pero no más allá, por ejemplo, facilitando las oportunidades de trabajar en los procesos de creación de comunidades de aprendizaje.

En líneas generales, tanto las familias como el alumnado y profesorado participante en este estudio valoran positivamente el uso de las TIC y sus posibilidades en el ámbito familiar y escolar, siendo mayores las ventajas identificadas frente a un escaso número de dificultades encontradas, como son los riesgos de internet, lo que concuerda con los resultados en investigaciones previas (Gómez, Llorente \& Morales, 2019). Sin embargo, aunque tanto las familias como el alumnado valoran que la dotación del centro y el uso de la tecnología que hace el profesorado es adecuada, una vez más la clave parece estar en la CD del docente (Peirats, Marín, Granados \& Morote, 2018) y en la introducción de innovación en la escuela. Un mero cambio de soporte no es suficiente para producir el cambio necesario en la educación del siglo XXI (Hoogerwerf, Solander-Gross, Mavrou, Traina \& Hersh, 2017; McDougall, Readman \& Wilkinson, 2018). La adquisición de CD para las familias en la mayoría de los centros se vincula a la mejora de las posibilidades futuras de inserción laboral, coincidiendo con los resultados del estudio de caso de Martínez-Piñeiro, Vila \& Gewerc (2018), pero ignorando las posibilidades de enriquecimiento que supone la interacción con otras personas, lo cual no se recogen en el presente estudio.

En general, familia, profesorado y alumnado consideran positiva la integración de las TIC y la adquisición de $\mathrm{CD}$ en la escuela, con el matiz de los adultos, que la vinculan a las exigencias del futuro y al mercado laboral. También es unánime el reconocimiento de que esta integración no siempre implica adquisición de $\mathrm{CD}$, y no se debe ignorar la asociación que hacen miembros de los tres colectivos con el carácter innovador y motivador que impregnan al trabajo en la escuela. Todavía no se aprecia un discurso claro y unánime entre representantes del profesorado, las familias y el alumnado, sobre el potencial pedagógico de las TIC en el aula. De las cuatro escuelas, familias y parte del profesorado tienen una desigual implicación en la implantación de las TIC y en el desarrollo de proyectos dirigidos a la adquisición de la $\mathrm{CD}$ que facilite una mayor inclusión educativa y social, y, que contribuya a abordar problemas individuales y colectivos. Con ciertos matices, todos los agentes identifican la importancia del uso de dispositivos digitales, la adquisición de la alfabetización que pueda contribuir a acceder y facilitar la comprensión y la asimilación de contenidos tradicionales asociados a la escuela y la comunicación con poseer CD. Los adultos reflejan su preocupación por el uso responsable y seguro de la tecnología, aunque son puntuales las iniciativas de los centros para trabajar específicamente esta cuestión.

Los colegios estudiados se ubican en contextos socioeconómicos y culturales diferentes, pertenecientes a dos comunidades autónomas, tres de ellos son públicos y uno concertado, dos se sitúan en el ámbito rural y otros dos en el urbano, unos desarrollan sus propios proyectos de integración de las TIC y otros, se acogen a las iniciativas de las administraciones autonómicas para la digitalización de la escuela. Sin embargo, como se ha venido explicando, existen importantes coincidencias en los resultados obtenidos: la integración de las TIC ya está en los centros, pero todavía no existe un discurso consolidado sobre las aportaciones de la $\mathrm{CD}$ al desarrollo educativo y social del alumnado dentro de la sociedad del conocimiento y de la información. En general, los centros

Competencia digital e inclusión educativa. Visiones de profesorado, alumnado y familias. $\mathrm{M}^{\mathrm{a}}$ Montserrat Castro Rodríguez, Diana Marín Suelves y Héctor Saiz Fernández.

Página 28 de 37 
presentan dificultades para implementar proyectos educativos coherentes, consistentes, permanentes en el tiempo, que contribuyan a adquirir una CD compleja y más completa. Alguna de las comunidades educativas, ha encontrado en las TIC una posibilidad para superar o reducir la brecha social, favoreciéndoles su inclusión en la sociedad en general, proyectándose y haciéndose visible en ella, aportando creación de conocimiento, aunque a veces implique cesiones a la inclusión escolar; frente comunidades que han optado por modelos más centrados en la CD dentro de la escuela y priorizando la inclusión educativa.

La clave del éxito en el proceso de integración de las tecnologías en la escuela, se encuentra en la construcción desde un modelo pedagógico claro que permita, además del desarrollo de la CD (Jasiewicz, Mierzecka y Kisilowska, 2018), la adquisición de habilidades para la vida y la inclusión en un sentido amplio.

Los retos en un futuro próximo se encuentran en la superación de las dificultades señaladas por los participantes en este estudio, como pueden ser la comunicación del centro con y entre las familias, tal y como señalaron Waliño, Pardo, Esnaola y San Martín (2018) o la conectividad. Por otra parte, los dispositivos digitales son utilizados diariamente y en la mayoría de materias. Sin embargo, es común que el uso de los dispositivos varíe en función de la asignatura, centrándose unas más en el trabajo con tecnología y otras menos. En general, Educación Física y Música son las dos materias donde menos uso se hace de los dispositivos y los materiales didácticos digitales, por tanto, esta es otra cuestión pendiente. Parece evidente también que es necesario aumentar la investigación en el campo de la inclusión social y educativa de personas con diversas características, capacidades y realidades, en cuanto al aprendizaje mediado por tecnología, a pesar de que en los últimos tiempos han aumentado los estudios, por ejemplo, centrados en alumnado con TEA (Saladino, Marín y San Martín, 2019) o discapacidad intelectual (Vidal, López, Marín y Peirats, 2018). Por otra parte, queda pendiente el análisis de cómo y quiénes construyen los libros de texto digitales y otros materiales didácticos digitales para la enseñanza (Knudsen y Selander, 2019).

A pesar que las limitaciones derivadas del tamaño de la muestra de este estudio no permiten la generalización de los resultados, se considera que son centros que reflejan diferentes contextos de nuestro país y que apuestan por la integración de las tecnologías en la escuela. Los resultados de este estudio permiten abrir nuevas líneas de investigación como el análisis de la realidad en otras etapas educativas como Infantil o Secundaria. También sobre la existencia o no de beneficios para el alumnado en cuanto a rendimiento, motivación y reducción del absentismo, así como sobre las implicaciones negativas que puede tener el uso de tecnología en el desarrollo personal y social del alumnado.

En definitiva, los cuatro centros estudiados se encuentran en contextos de aprendizaje donde la $\mathrm{CD}$ es reconocida en diferentes grados por su valor pedagógico y por sus contribuciones al desarrollo, tanto individual como colectivo. Las diferencias encontradas en lo que entienden por $\mathrm{CD}$, desde la instrumentalización hasta el uso crítico y pedagógico de tecnología, puede ser la razón de la diversidad en los grados de integración observados. Se relaciona también con que el uso de tecnología en el aula no siempre esté vinculado a un cambio metodológico o a metodologías más activas que permitan un alto grado de integración de la tecnología en el proceso de enseñanza-aprendizaje y, a su vez, un auténtico desarrollo de la $\mathrm{CD}$, tanto en los docentes como del alumnado. Parece evidente 
la necesidad de nuevas políticas para la formación del profesorado para adquirir una mayor CD para plasmar en la labor pedagógica (Pérez Escoda y Rodríguez Conde, 2016).

En conclusión, es fundamental trabajar por y para la inclusión digital, que es definida por Luque (2012), como la participación plena de todos los ciudadanos, en igualdad de condiciones, en la Sociedad del Conocimiento, garantizando tanto el acceso a las tecnologías como su uso. Para ello, en las escuelas del siglo XXI se debería redefinir el concepto de alfabetización y de ciudadanía (Area, 2019).

Presentación del artículo: 23 de octubre de 2019 Fecha de aprobación: 11 de noviembre de 2019 Fecha de publicación: 30 de noviembre de 2019

Castro, M., Marín, D. \& Sáiz, H. (2019). Competencia digital e inclusión educativa. Visiones de profesorado, alumnado y familias. RED. Revista Educación a Distancia, 61. DOI: http://dx.doi.org/10.6018/red/61/06

\section{Financiación}

El Proyecto de Investigación "Competencia digital en estudiantes de educación obligatoria. Entornos sociofamiliares, procesos de apropiación y propuestas de einclusión" (CDEPI) está financiado por el Ministerio de Economía y Competitividad (EDU2015-67975-C3-1-P) y por el Fondo Europeo de Desarrollo Regional (FEDER). Los hallazgos que se presentan en este artículo corresponden al estudio III cuyo objetivo fundamental se centra en analizar el uso educativo de materiales y contenidos digitales en las aulas, así como su relevancia en relación al proceso de enseñanza aprendizaje y cómo influye este en la experiencia educativa del alumnado.

\section{Referencias}

Alba, C. (2016). Diseño Universal para el Aprendizaje: educación para todos y prácticas de enseñanza inclusivas. Madrid: Editorial Morata.

Anggeraini, Y., Faridi, A., Mujiyanto, J. \& Bharati, D.A.L. (2019). The teachers' perceptions on digital literacy competences in EFL classroom. Asian EFL Journal, 24(4), 5-12.

Area, M. \& Hernández, V. (2018). La escuela digital: Análisis de las representaciones y prácticas de los actores educativos ante los materiales didácticos en la Red. Recuperado de http://bit.ly/32xBbRg

Area, M. (2006). Veinte años de políticas institucionales para incorporar las tecnologías de la información y comunicacional sistema escolar. En J. M ${ }^{a}$ Sancho (coord.), Tecnologías para transformar la educación. Madrid: AKAL/UIA.

Area, M. (2017). La metamorfosis digital del material didáctico tras el paréntesis Gutenberg. RELATEC, 16(2), 13-28.

Area, M. (2019). Reinventing schools and the educational materials in the digital society. En J. Rodríguez, T. M. Braga y E. Bruillard (EDs.), IARTEM 1991-2016: 25 years 
developing textbook and educational media research. Santiago de Compostela: Andavira Editoral S.L.

Area, M., Hernández, V.M. \& Sosa, J.J. (2016). Modelos de integración didáctica de las TIC en el aula. Comunicar: Revista científica iberoamericana de comunicación y educación, (47), 79-87.

Area, M., Sanabria, A. L. \& Santana, P. J. (2018). La transformación digital del sistema escolar público en Canarias: informe dirigido al Consejo Escolar de Canarias. Recuperado de http://bit.ly/31taAn4

Arnáiz, P. (2012). Escuelas eficaces e inclusivas: cómo favorecer su desarrollo. Educatio Siglo XXI, 30(1), 25-44.

Azorín, C. M. \& Arnáiz, P. (2013) Tecnología digital para la atención a la diversidad y mejora educativa.Etic@net, 1(13), 14-29.

Bender, W., Urrea, C. \& Zapata-Ros M. (2015). Presentación. RED-Revista de Educación a Distancia, 46 (3), 1-3. doi: 10.6018/red/46/1

Bogdan, R.C. \& Biklen, S.K. (1982). Qualitative research for education: an introduction to theory and methods. Londres: Allyn and Bacon.

Cabañes Martínez, E. (2016). La tecnología en las fronteras (Tesis doctoral). Madrid, Universidad Autónoma.

Cabero, J. (2004). Reflexiones sobre la brecha digital y la educación. En F.J. Soto y J. Rodríguez (coords.), Tecnología, educación y diversidad: retos y realidades de la inclusión social (pp. 23-42). Murcia: Consejería de Educación y Cultura.

Camacho Martí, M.M. (2007). Theacher Training In ICT-Based Learning Settings: Desing And Implementation Of An On-Line Instructional Model For English Language Teachers (Tesis doctoral). Tarragona, Universitat Rovira i Virgili.

CAST (2011). Universal Design for Learning Guidelines version 2.0. Wakefield, MA. Recuperado de http://bit.ly/2P1fzIU

Castells, M. (1998). Paraísos comunales: identidad y sentido en la sociedad red. La era de la información. Economía, sociedad y cultura, 2, 27-90.

Castro, M., Rodríguez, J. \& Peirats, J. (2017). Materiales Didácticos, Libros de Texto y Educación Inclusiva. Educatio siglo XXI, 35(3), 11-16. Recuperado de http://ir.uv.es/UFpIPk0

Céspedes, R. \& Ballesta, J. (2018). Acceso, uso y actitud de la tecnología en las escuelas de Educación Primaria. Aula Abierta, 47(3), 355-364.

Cid Fernández, X.M. \& Rodríguez Rodríguez, J. (coord.) (2007). A fenda dixital e as súas implicacións educativas. Santiago: Nova Escola Galega.

Colas, M.P., De Pablos, J. \& Ballesta, J. (2018). Incidencia de las TIC en la enseñanza en el sistema educativo español: una revisión de la investigación. RED. Revista de Educación a Distancia, 56. Recuperado de http://bit.ly/2OXMWMR

Cook, T.D. \& Campbell, D.T. (1979). Quasi-experimention: Design and Analysis Issues for Field Settings. Chicago: Rand McNally. 
Darcy, S., Yerbury, H. \& Maxwell, H. (2019). Disability citizenship and digital capital: the case of engagement with a social enterprise telco. Information Communication and Society, 22(4), 538-553.

De Almeida, M.E.B., Teixeira, A.R.A. \& Almeida, R. (2019). Work in progress: Improving learning performance using programming methodology. IEEE Global Engineering Education Conference, EDUCON, 1462-1466

De Pablos, J., Colás, M. P., Conde, J. \& Reyes, S. (2016). La competencia digital de los estudiantes de educación no universitaria: variables predictivas. Bordón, 68 (2), $1-17$.

De Pablos, J., Colás, P. \& González, T. (2010). Factores facilitadores de la innovación con TIC en los centros escolares. Un análisis comparativo entre diferentes políticas educativas autonómicas. Revista de Educación, 352, 23-51.

Del Moral, M. E., Villalutre, L. \& Neira, M. R. (2014). Variables asociadas a la cultura innovadora con TIC en escuelas rurales. Profesorado. Revista de Currículum y Formación de Profesorado, 18(3), 9-25.

Delfino, G. I., Beramendi, M. \& Zubieta, E. (2019). Participación social y política en Internet y brecha generacional. Revista de Psicología, 37(1), 195-216.

Etchegaray, M.C., Guzmán, M.D. \& Duarte, A.M. (2017). Diseño de un recurso multimedia on line basado en Inteligencias Múltiples. Campus Virtuales, 6(1), 5165.

Fernández, F.J., Fernández, M.J. \& Rodríguez, J.M.R. (2018). El proceso de integración y uso pedagógico de las TIC en los centros educativos madrileños. Educación XX1, 21(2), 395-416. doi: 10.5944/educXX1.17907.

Flick, U. (2018). An introduction to qualitative research. London: Sage.

Fraga, F. \& Rodríguez, A. (2019). La Competencia Digital ante contextos de exclusión: un estudio de caso en Educación Primaria. Revista Latinoamericana de Tecnología Educativa, 18(1), 55-70. Recuperado de http://bit.ly/2Brcfij

Fuentes, A., López, J. \& Pozo, S. (2019). Análisis de la Competencia Digital Docente: Factor Clave en el Desempeño de Pedagogías Activas con Realidad Aumentada. REICE. Revista Iberoamericana sobre Calidad, Eficacia y Cambio en Educación 17(2), 27-42.

Gamito, R., Aristizabal, P. \& Vizcarra, M. T. (2019). Sociedad multipantalla: un reto educativo para familia y escuela. Revista Prisma Social, (25), 398-423.

Gewerc, A. \& Fraga-Varela, F. (2019). Competencia digital e inclusión social: cuando las condiciones ocioculturales se imponen. Gewerc, A. \& Martínez-Piñeiro, E. Competencia digital y preadolescencia. Los desafíos de la e-inclusión. Madrid: Síntesis, 21-42.

Gewerc, A. \& Martínez-Piñeiro, E. (2019). Competencia digital y preadolescencia. Los desafíos de la e-inclusión. Madrid: Síntesis.

Gewerc, A., \& Montero, L. (2015). Conocimiento profesional y competencia digital en la formación del profesorado. El caso del Grado de Maestro en Educación Primaria. RELATEC, 14 (1), 31-43. Recuperado de http://bit.ly/2K6CVtf 
Gonçalves, D., Fernández, J., Castro, M., Ricoy, M.C., Rodríguez, X. \& Cid, X.M. (2018). A Fenda Dixital: TIC, NEAE, Inclusión e Equidade. En IV Congresso Internacional" A Fenda DIgital". Porto: Escola Superior de Educação de Paula Frassinetti (ESEPF).

González Pérez, A. (2010). ¿Qué nos interesa evaluar de las políticas educativas TIC españolas? Revista Fuentes, 10, 206-220.

González Pérez, A. (2011). Evaluación del impacto de las políticas educativas TIC en las prácticas de los Centros Escolares (tesis doctoral). Sevilla: Universidad de Sevilla.

González, C. J., Martín, S. \& Vega, A. (2018). Portales educativos: la producción de materiales didácticos digitales. @tic revista d'innovació educativa, 20, 89-97.

Gutiérrez, A. \& Tiner, K. (2012). Educación para los medios, alfabetización mediática y competencia digital. Comunicar, 38(XIX), 31-39.

Hernández-Sánchez, A., Quijano, R. \& Pérez, M. (2019). La formación digital del estudiante universitario digital: competencias, necesidades y pautas de actuación. Hamut'ay, 6(1), 19-32. doi: http://dx.doi.org/10.21503/hamu.v6i1.1572

Holgado, C. (2016). Secuenciación de unidades didácticas: propuesta metodológica para lenguas modernas como alternativa a los EVA. Campus Virtuales, 5(2), 84-98.

Hoogerwerf, E.-J., Solander-Gross, A., Mavrou, K., Traina, I. \& Hersh, M. (2017). A Self-Assessment Framework for Inclusive Schools Supporting Assistive Technology Users. Studies in Health Technology and Informatics, 242, 820-827.

Hsin, C.T., Li, M.C. \& Tsai, C.C. (2014). The influence of young children's use of technology on their learning: A review. Educational Technology and Society, 17(4), 85-99.

INTEF (2017). Marco Común de la Competencia Digital Docente. Madrid: Instituto Nacional de Tecnologías Educativas y Formación del Profesorado Ministerio de Educación, Cultura y Deporte. Recuperado de http://bit.ly/2qoyP8R

Jasiewicz, J., Mierzecka, A. \& Kisilowska, M (2018). Complex and Multivariable: Methodology of Exploring Digital Literacy and Training Needs Within the Polish SME Sector. Communications in Computer and Information Science, 810, 322331.

Jódar Marín, J.Á. (2010). La era digital: nuevos medios, nuevos usuarios y nuevos profesionales. Razón y palabra, 15(71). Recuperado de http://bit.ly/2MVlkVT

Juhaňák, L., Zounek, J., Záleská, K., Bárta, O. \& Vlčková, K. (2019). The relationship between the age at first computer use and students' perceived competence and autonomy in ICT usage: A mediation analysis. Computers and Education, 141,103614

Knudsen, S.V. \& Selander, S. (2019). Learning resourcesat stake. Main trends and new challenges in research on textbooks and educational media. En J. Rodríguez, T. M. Braga y E. Bruillard (EDs.) IARTEM 1991-2016: 25 years developing textbook and educational media research. Santiago de Compostela: Andavira Editoral S.L.

Kvale, S. (2011). Las entrevistas en investigación cualitativa. Madrid: Morata. 
Ley Orgánica 8/2013, de 9 de diciembre, para la Mejora de la Calidad Educativa (LOMCE). Boletín Oficial del Estado n²95 de 10 de diciembre de 2013. Recuperado de http://bit.ly/2VW2mCv

Lloyd-Jones, G. (2003). Design and control issues in qualitative case study research. International Journal of Qualitative Methods, 2(2).

Lores Gómez, B. (2017). Estudio descriptivo del uso de la tic en educación primaria como respuesta a la realidad educativa y social en la provincia de Castellón (Tesis doctoral).Universidad CEU, Castellón.

Losada, D., Correa, J.M. \& Fernández, L. (2017). El impacto del modelo «un ordenador por niño» en la Educación Primaria: Un estudio de caso. Educación XX1, 20(1), 339-361. doi: 10.5944/educXX1.17515.

Lozano, J.; Castillo, I. S. \& Cerezo, M. C (2014). ¿Es posible enseñar a las personas con TEA habilidades emocionales y sociales y generalizar esos aprendizajes a otros contextos?: un estudio de caso en un centro de Educación Primaria. Didáctica, innovación y multimedia, 30, 1-14. https://ddd.uab.cat/record/131900

Luque, A. (2012). La educación inclusiva y el mundo digital: nuevos retos en la sociedad del conocimiento.Etic@net, 2(12),202-215.

Macià, M. \& Garreta, J. (2018). Accesibilidad y alfabetización digital: barreras para la integración de las TIC en la comunicación familia/escuela. Revista de Investigación Educativa, 36(1), 239-257. DOI: http://dx.doi.org/10.6018/rie.36.1.290111

Maquilón Sánchez, J.J. (2016). Relación entre el pensamiento compuatacional y el rendimiento académico. EDUNOVATIC 2016 - I Congreso Virtual internacional de Educación, Innovación y TIC, 694-697.

Marín, D., Morote, D., \& Aguasanta, M. (2019). Diversidad y tecnología: el blog de centro como herramienta para la inclusión En Ramírez Paredes, K.G. (Coord.), Recursos educativos para el aula del siglo XXI (pp. 1-10). Eindhoven, NL: Adaya Press.

Marjories, Y., Campaña, R.L. \& Gallego, M.J. (2018). Iniciativas para la adopción y uso de recursos educativos abiertos en Instituciones de Educación Superior. Revista Cubana de Educación Médica Superior, 32(4), 273-285.

Martínez-Piñeiro, E., Vila, E. \& Gewerc, A. (2018). El papel de la familia en la construcción de la competencia digital. RISTI-Revista Ibérica de Sistemas e Tecnologias de Informação, 28, 1-13.

Martínez-Serrano, M. C. (2019). Percepción de la Integración y uso de las Tecnologías de la Información y la Comunicación (TIC). Estudio de Profesores y Estudiantes de Educación Primaria. Información tecnológica, 30(1), 237-246.

McDougall, J., Readman, M. \& Wilkinson, P. (2018). The uses of (digital) literacy. Learning, Media and Technology, 43(3), 263.

Merriam, S.B. (1988). Case Study research in education. A Qualitative Approach. San Francisco: Jossey-Bass.

Miles, M., Huberman, M. \& Saldaña, J. (2014). Qualitative data analysis: a methods sourcebook. Londres: Sage Publications. 
Millard, A. Baldassar, L. \& Wildingb, R. (2018). The significance of digital citizenship in the well-being of older migrants. Public Health, 158, 144-148.

Musmarra, P. (2017). The safety of your own app with app inventor. Advances in Intelligent Systems and Computing. 11th International Conference on Complex, Intelligent, and Software Intensive Systems, CISIS, 611, 1037-1043.

Ojeda-Castelo, J.J., Piedra-Fernandez, J.A., Iribarne, L. \& Bernal-Bravo, C. (2018). KiNEEt: application for learning and rehabilitation in special educational needs. Multimedia Tools and Applications, 77 (18), 24013-24039

Orden ECD/65/2015, de 21 de enero, por la que se describen las relaciones entre las competencias, los contenidos y los criterios de evaluación de la educación primaria, la educación secundaria obligatoria y el bachillerato.

Peirats, J., Gabaldón, D. \& Marín, D. (2018). Percepciones sobre materiales didácticos y la formación en competencia digital. @ tic revista d'innovació educativa, 20, 5462.

Peirats, J., Gallardo, I. M., San Martín, Á. \& Waliño, M.J (2016). Análisis de la industria editorial y protocolo para la selección del libro de texto en formato digital. Profesorado. Revista de Currículum y Formación de Profesorado, 20(1), 76-90.

Peirats, J., Marín, D., Granados, J. \& Morote, D. (2018). Competencia digital en los planes de estudio de universidades públicas españolas. REDU. Revista de Docencia Universitaria, 16(1), 175-191.

Pérez Escoda, A. \& Rodríguez Conde, M.J. (2016). Evaluación de las competencias digitales autopercibidas del profesorado de educación primaria en Castilla y León. Revista de Investigación Educativa, 34(2), 399-415. doi: http://dx.doi.org/10.6018/rie.34.2.215121

Pérez, A., Castro, A. \& Fandos, M. (2016). La competencia digital de la Generación Z: claves para su introducción curricular en la Educación Primaria. Comunicar: Revista científica iberoamericana de comunicación y educación, 49, 71-79.

Pérez-Escoda, A. \& Fernández-Villavicencio, N.G. (2016). Digital competence in use: From DigComp 1 to DigComp 2. ACM International Conference Proceeding Series, 619-624.

Perryman, C. L., \& Jeng, L. H. (2019). Changing Models of Library Education to Benefit Rural Communities. Public Library Quarterly. doi: 10.1080/01616846.2019.1621736

Pötzsch, H. (2016). Materialist perspectives on digital technologies: Informing debates on digital literacy and competence. Nordicom Review, 37(1), 119-132.

Prensky, M. (2001). Digital natives, digital immigrants part 1. On the horizon, 9(5), 1-6.

Price-Dennis, D., Holmes, K.A. \& Smith, E. (2015). Exploring digital literacy practices in an inclusive classroom. Reading Teacher, 69(2), 195-205.

Ramírez-García, A., Díaz, V. \& Sánchez-Carrero, J. (2014). ¿Sabes más que un niño de Primaria? La competencia mediática del alumnado de $4^{\circ}$ de Educación Primaria en Andalucía. Revista Complutense De Educación, 25(2), 293-312. doi: https://doi.org/10.5209/rev_RCED.2014.v25.n2.41531. 
Recomendación del Parlamento Europeo y el Consejo de 18 de diciembre de 2006 sobre las competencias clave para el aprendizaje permanente (2006/962/CE). L 394/10. Diario Oficial de la Unión Europea. Recuperado de http://goo.gl/3wsA98

Rego, L. \& Marín, D. (2019). Las visiones del alumnado sobre los Materiales Didácticos Digitales en España. Educar em revista, 35(77), 79-94.

Reyero, M. (2019). La educación constructivista en la era digital. Revista Tecnología, Ciencia y Educación, 12, 111-127.

Roig-Vila, R., Lorenzo-Lledó, A. \& Mengual-Andrés, S. (2019). Utilidad percibida de la realidad aumentada como recurso didáctico en Educación Infantil. Campus Virtuales, 8(1), 19-35.

Saladino, M., Marín, D. \& San Martín, A. (2019). Aprendizaje mediado por tecnología en alumnado con tea. Una revisión bibliográfica.Etic@ net, 1, 1-25.

Sanabria, A., Álvarez, Q. \& Peirats, J. (2017). Las políticas educativas en la producción y distribución de materiales didácticos digitales. RELATEC, 16(2), 63-77. doi: https://doi.org/10.17398/1695288X.16.2.63.

Sanabria, A.L. \& Cepeda, O. (2016). La educación para la competencia digital en los centros escolares: la ciudadanía digital. RELATEC: Revista Latinoamericana de Tecnología Educativa, 15(2), 95-112. doi: https://doi.org/10.17398/1695288X.15.2.95.

Sánchez Montoya, R. (2007). Las tecnologías en la Escuela Inclusiva: nuevos escenarios, nuevas oportunidades. Conclusiones $4^{\circ}$ Congreso de Tecnología Educativa y Atención a la Diversidad, Tecnoneet, y al $6^{\circ}$ Congreso Iberoamericano de Informática Educativa Especial, CIIEE. Didáctica, Innovación y Mutimedia, 9, 110. Recuperado de http://bit.ly/32tvXpq

Sánchez, J.M. \& Arathoon, A.I. (2016). Recursos digitales y Diseño Universal para el Aprendizaje. En Alba, C. (Coord.), Diseño Universal para el Aprendizaje: Educación para todos y prácticas de enseñanza inclusivas (89-122). Madrid: Morata.

Sánchez, J.M., Alba, C. \& Sánchez, P. (2018). Valoraciones del alumnado de Educación Primaria sobre lecturas digitales diseñadas con UDL Book-Builder como apoyo en los procesos lectores. Aula abierta, 47(4), 481-490.

Sancho-Gil, J. M. \& Hernández-Hernández, F. (2018). La profesión docente en la era del exceso de información y la falta de sentido. Revista de Educación a Distancia, 56, 1-23. doi: http://dx.doi.org/10.6018/red/56/4

Santana, P. J., Eirín, R. \& Marín, D. (2017). Análisis y evaluación de portales institucionales en España. Los casos de Canarias, Galicia y Valencia. Revista Latinoamericana de Tecnología Educativa-Relatec, 16(2), 29-48.

Sanz, J.J. (2017). Percepciones y valoraciones de la utilización de los libros digitales en Educación Superior. Campus Virtuales, 6(1), 39-50.

Selwood, A., Atkinson, J. \& Black, R. (2005). Bridging the digital divide: An analysis of a notebook borrowing program at a rural primary school in Australia. Proceedings of the Fourth IASTED International Conference on Communications, Internet, and Information Technology, 266-273. 
Severín, E., Peirano, C. \& Falck, D. (2012). Guía básica para la evaluación de proyectos: tecnologías para la Educación. Banco Interamericano de Desarrollo. Recuperado de http://bit.ly/2JJkZWw

Spante, M. (2019). Digital creativity: learning by story driven digital production. International Journal of Information and Learning Technology, 36, 182-191.

Stake, R. (1998). Investigación con estudio de casos. Madrid: Morata.

Steck, C. (2018). Una digitalización sostenible, inclusiva y justa. Política Exterior, 32 (185), 58-62.

Tárraga, R., Vélez-Calvo, X., Lacruz-Pérez, I., \& Sanz-Cervera, P. (2019). Efectividad del uso de las TIC en la intervención educativa con estudiantes con TEA. DIM: Didáctica, Innovación y Multimedia, 37, 1-10.

Tedesco, J.C. (2005). Los pilares de la educación del futuro. Revista Colombiana de Sociología, 25, 11-23.

Umar, I.N. \& Jalil, N. (2012). ICT skills, practices and barriers of its use among secondary school students. Procedia-Social and Behavioral Sciences, 46, 5672-5676.

Valverde, J. \& Sosa, M.J. (2015). El modelo de un ordenador por alumno en Centros de Educación Primaria. Prácticas educativas y organización escolar en un estudio de caso múltiple. En J. De Pablos (Coord.), Los centros educativos ante el desafío de las tecnologías digitales (pp.119-173). Madrid, España: La Muralla.

Valverde, J., Fernández, M. R. \& Garrido, M. C. (2015). El pensamiento computacional y las nuevas ecologías del aprendizaje. Revista de Educación a Distancia, 46(3), 1-18. 1-18. doi: $10.6018 / \mathrm{red} / 46 / 3$

Van de Oudeweetering, K. \& Voogt, J. (2018). Teachers' conceptualization and enactment of twenty-first century competences: exploring dimensions for new curricula. Curriculum Journal, 29(1), 116-133.

Vidal, M. I., López, M., Marín, D. \& Peirats, J. (2018). Revisión y análisis de investigación publicada sobre intervención gamificada en discapacidad intelectual. Revista científica electrónica de Educación y Comunicación en la Sociedad del Conocimiento, 2(18), 274-297.

Vuorikari, R., Punie, Y., Carretero, S. \& Van den Brande, G. (2016). DigComp 2.0: The Digital Competence Framework for Citizens. Update Phase 1: The Conceptual Reference Model. Luxembourg Publication Office of the European Union. EUR 27948 EN. doi:10.2791/11517

Waliño, M. J., Pardo, M. I., Esnaola, G. E. \& San Martin, Á. (2018). La participación escolar de las familias a través de plataformas digitales. @ tic. revista d'innovació educativa, 20, 80-88. doi: https://doi.org/10.7203/attic.20.12131

Yin, R.K. (2003). Case study research (3rd ed.). Thousand Oaks, CA: Sage.

Zhang, H. \& Zhu, C. (2016). A Study of Digital Media Literacy of the 5th and 6th Grade Primary Students in Beijing. Asia-Pacific Educational Research, 25(4), 579-592. 